\title{
Nanotribological studies using nanoparticle manipulation: Principles and application to structural lubricity
}

\author{
Dirk DIETZEL ${ }^{1, *}$, Udo D. SCHWARZ ${ }^{2}$, André SCHIRMEISEN ${ }^{1}$ \\ ${ }^{1}$ Institute of Applied Physics (IAP), Justus-Liebig-Universität Giessen, Germany \\ ${ }^{2}$ Departments of Mechanical Engineering \& Materials Science and Chemical \& Environmental Engineering and Center for Research \\ on Structures and Phenomena (CRISP), Yale University, New Haven, CT, USA
}

Received: 13 April 2014 / Accepted: 02 June 2014

(C) The author(s) 2014. This article is published with open access at Springerlink.com

\begin{abstract}
The term "structural lubricity" denotes a fundamental concept where the friction between two atomically flat surfaces is reduced due to lattice mismatch at the interface. Under favorable circumstances, its effect may cause a contact to experience ultra-low friction, which is why it is also referred to as "superlubricity". While the basic principle is intriguingly simple, the experimental analysis of structural lubricity has been challenging. One of the main reasons for this predicament is that the tool most frequently used in nanotribology, the friction force microscope, is not well suited to analyse the friction of extended nanocontacts. To overcome this deficiency, substantial efforts have been directed in recent years towards establishing nanoparticle manipulation techniques, where the friction of nanoparticles sliding on a substrate is measured, as an alternative approach to nanotribological research. By choosing appropriate nanoparticles and substrates, interfaces exhibiting the characteristics needed for the occurrence of structural lubricity can be created. As a consequence, nanoparticle manipulation experiments such as in this review represent a unique opportunity to study the physical conditions and processes necessary to establish structural lubricity, thereby opening a path to exploit this effect in technological applications.
\end{abstract}

Keywords: Nanotribology; nanoparticle manipulation; friction force microscopy; structural lubricity; superlubricity; HOPG

\section{Nanoparticle manipulation: An alternative route to nanotribology}

Due to the continuing miniaturization of micromachinery, such as found in nano-electromechanical systems [1, 2], considerable research efforts are directed towards the understanding and optimization of frictional behaviour on the nanometer scale. Nanoscale systems that include moving parts comprise a considerable challenge for scientists and engineers since functionality and durability are often limited by the effects of friction and wear. Conventional approaches to manage friction and wear, such as lubrication or surface modifications, are, however, difficult to apply

* Corresponding author: Dirk DIETZEL.

E-mail: dirk.dietzel@ap.physik.uni-giessen.de for small-scale systems, and other ways to control friction need to be established. An indispensable prerequisite for substantive progress is to first gain detailed understanding of tribological processes at the micro- and nanoscale. As a consequence, the field of nanotribology [3, 4], which explores the fundamentals of friction on the nanometer scale, has been constantly growing during the last 20 years.

One of the most important experimental tools for nanotribological studies is the friction force microscope (FFM), which was introduced in 1987 by Mate et al. [5]. Friction force microscopy expands the capabilities of a conventional atomic force microscope (AFM) [6] by introducing a position-sensitive photodiode that detects the torsion of a cantilever, which is the forcesensing device in an atomic force microscope, simultaneously to the cantilever's normal displacement. 
Since the torsion of the cantilever is induced by the forces acting on a sharp tip located at the end of the cantilever while the tip is sliding over a surface, the quantification of the torsion as a function of the local position, applied normal force, and sliding speed allows to analyse tribological processes at the nanoscale $[7,8]$. If measuring friction in this configuration, the contact between tip and sample can be viewed as a single asperity contact, which represents the classical model geometry when it comes to develop an understanding of the microscopic origins of friction. This approach is inspired by the widely accepted theories of Bowden and Tabor [9], who suggested that any real surface can be approximated by a collection of asperities. A single asperity contact can thus be regarded as the fundamental building block of friction as it is illustrated in Ref. [10].

Applying friction force microscopy as an experimental technique has enabled researchers to directly explore the elementary processes that give rise to the occurrence of friction at nanoscale point contacts. For example, measurements have been performed for a large number of sample systems while systematically varying experimental parameters such as the normal force applied by the cantilever [11-16], the radius of the AFM tip [11, 13, 14], the sliding velocity [17-20], the sample temperature [21-23], the relative orientation between scan direction and substrate lattice [24-27], or the chemical nature of the sample [28-30]. But despite the many successes, the experimental configuration realized in an FFM also has inherent limitations that impede the application of friction force microscopy to an even wider range of tribological questions. The most significant constraints are:

1. Material combinations: While there are no constraints regarding the choice of samples under investigations, experimentalists face limited availability of materials for AFM tips, which mostly consist of $\mathrm{Si}$, $\mathrm{SiO}, \mathrm{SiN}$ or diamond. The spectrum of material combinations can be increased by using coated tips. Such tips are, however, often characterized by poor quality since both the tip's geometry as well as its atomic structure are usually ill defined, which makes a reliable interpretation of experimental results difficult. Furthermore, coated tips are prone to degradation by wear, since many coatings attach only weakly to the tips (e.g., gold layers on silicon). This is especially problematic for systematic studies, which require multiple scans with varying parameters.

2. Structure: Since the apex of most AFM tips is disordered, it is very difficult to analyze the effects of local atomic order, such as exhibited by crystalline surfaces sliding over each other, on interfacial friction. The study of the friction experienced by ordered interfaces is, on the other hand, becoming an increasingly interesting topic since such interfaces can exhibit drastically altered friction values [31]. Scientific interest is mostly focused onto an effect often denoted as either superlubricity or, more adequately, structural lubricity, where ultralow friction can be achieved due to a structural mismatch between the two interfaces in contact. A more detailed description of this effect will be provided in Section 3.

3. Contact area: Another fundamental question in nanotribology is related to the contact area dependence of friction. While macroscopic friction is considered to be independent of the contact area, the situation is less clear on the nanoscale. Recent theoretical results suggest that the contact area dependence of friction can sensitively depend on the precise interface structure, thereby relating its analysis closely to the previous point of ill-defined tip structures. On a more fundamental level, we note that it is generally difficult to quantify the exact contact area between an AFM tip and a surface since there is no routinely applicable method to measure the real contact area between AFM tip and sample. The situation is further complicated by the need to vary the contact area during an experiment if the contact area dependence of friction should be assessed. Small changes of the contact area can be achieved by increasing or decreasing the normal force exerted on the AFM tip, which may then be quantified by using contact mechanical models if well-defined tips are employed. Despite some successes [13, 14], this approach as of now failed to provide insight into many fundamental questions related to the contact area dependence of friction.

4. Shape: Recent theoretical analysis indicates that in the case of structural lubricity, interfacial friction is not only governed by the size of the contact area itself, but can also sensitively depend on the shape of the contact area [32,33]. For the contact formed by an AFM tip and a sample, the precise shape of the 
contact area is even more difficult to assess than it's size. Consequently, there are presently no friction force microscopy studies available that would relate interfacial friction to the shape of the contact area.

These shortcomings of friction force microscopy illustrate that many essential issues in nanotribology require an alternative approach if they should be addressed. Ideally, contacts of well-defined structure, shape, size and variable orientation need to be analysed. Such characteristics can be found with nanoparticles supported by flat surfaces, which can, e.g., be prepared by thermal evaporation. Depending on the material and preparation parameters, particles can either be crystalline or amorphous. Common choices for substrates include materials such as highly oriented pyrolytic graphite (HOPG) or $\mathrm{MoS}_{2}$ due to their easy-to-prepare surfaces featuring large atomically flat terraces, but comparatively weak particle-substrate interactions (van der Waals only).

Once a suitable sample system has been prepared, the motion of the nanoparticles on the substrate, needed to generate friction, can be induced by the tip of an atomic force microscope (see Fig. 1). The range of contact areas that is accessible by nanoparticle manipulation is indicated in Fig. 2 in comparison to
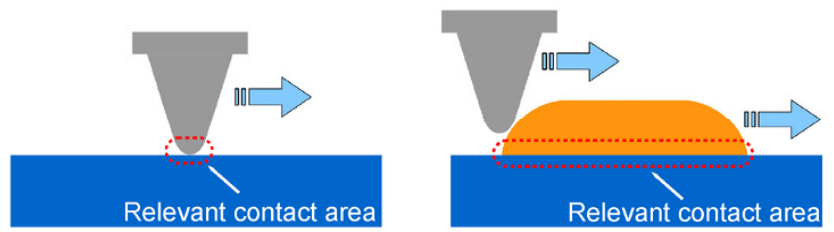

Fig. 1 The difference between conventional friction force microscopy studies and particle manipulation schemes lies in the relevant interface. While FFM is limited to friction occurring at the interface between tip and surface (left), the particle manipulation method allows to study the much larger, but well-defined particle/ surface interface (right).

Nanoscale

Macroscale

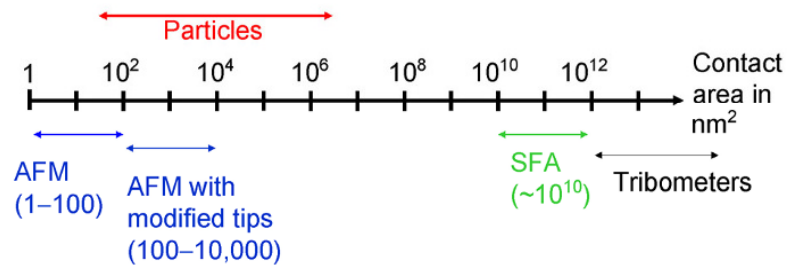

Fig. 2 Overview over the different ranges of contact areas covered by tribological techniques on the nano- and meso-scale. other experimental tools commonly used in nanoand microtribology, namely the friction force microscope (either with standard or modified tips) [5, 13, 14, 17], the surface force apparatus (SFA) [34, 35], and the quartz crystal microbalance (QCM) [36-38]. From the figure, we see that nanoparticle manipulation offers unique access to lengthscales at the transition between the nano- and meso-scale.

The concept of nanoparticle manipulation using atomic force microscopy was first demonstrated with the example of $\mathrm{C}_{60}$ islands grown on a $\mathrm{NaCl}$ surface [39] and later employed to investigate frictional anisotropies for $\mathrm{MoO}$ nanoparticles [40]. Nanoscale objects, like nanotubes, have been pushed to distinguish sliding and rolling motion [41]. Recently, there has been an increase of systematic friction studies using nanoparticle manipulation, highlighting the influence of surface structure on particle trajectories [42] as well as the influence of parameters like particle shape $[43,33]$, surface chemistry and temperature [44], and interface contamination [45-47]. The fundamental question of how friction is related to contact area has been addressed by a systematic variation of the size of metallic nanoparticles [33, 45, 48]. Furthermore, nanoparticle manipulation experiments have been used to analyze the difference between static and sliding friction. While some experimental approaches are mainly sensitive to either static friction [48, 49] or sliding friction [45], recent experiments have demonstrated how nanoparticle manipulation can be employed to measure static and sliding friction [50] or monitor the transition from static to sliding friction [51]. Very recently, the underlying physical phenomenon of the transition from static to sliding friction, i.e., contact ageing, could be analyzed by systematic temperature- and velocity-dependent nanomanipulation experiments [52]. This diversity of friction phenomena that can be analyzed by nanomanipulation experiments is due the large variety of tribological interfaces that can be created by combining particles and substrates that differ in size, geometry, structure, and chemical composition.

In many of the recent studies, a main objective is the analysis of frictional properties of very clean interfaces. To achieve this goal, it is mandatory to prepare nanoparticles under ultrahigh vacuum conditions (UHV) and transfer the samples to an 
UHV-AFM without breaking the vacuum, which is most commonly realized by the in-situ thermal evaporation of metals onto a flat substrate. Depending on the conditions during metal evaporation and particle growth nanoparticles of different shape and structure can be created.

For antimony nanoparticles on HOPG, a sample system that has frequently been used in the past, the precise growth parameters and corresponding atomic structures have been studied in detail by Stegemann et al. [53]. It was found that small, round shaped particles are usually amorphous, whereas larger particles are typically crystalline, an effect that strongly depends on the surface temperature during evaporation [54]. It is expected that nanoscale friction is governed by the atomistic structure of the particle-substrate interface, and indeed, Ritter et al. have recently reported on two distinct shear stresses observed during nanomanipulation experiments of antimony nanoparticles on HOPG under ambient conditions [55]. In other studies, gold nanoparticles produced from evaporation onto HOPG have been used, which showed a well defined crystalline structure structure. Comparing their frictional behavior to the one of similarly sized antimony nanoparticles allowed to directly assess the effect of atomic structure and order on friction [33].

Despite the potential for the analysis of fundamental effects in interfacial friciton, the approach of particle preparation by thermal evaporation is limited compared to chemical methods, which can yield a much higher variety of different shapes, sizes, and surface functionalization. For example, gold particles can be prepared in a wide range of geometries, including spheres, rods, and even star-like shapes [44]. Furthermore, they can be coated with self-assembled monolayers terminated with hydrophobic (e.g., methyl, $-\mathrm{CH}_{3}$ ) or hydrophilic groups (e.g., hydroxyl, $-\mathrm{OH}$ ). This allows to investigate the influence of the hydrophobicity of the coatings on the mobility of the nanoparticles [44]. For example, Tranvouez et al. have studied ligand-capped cadmium selenide nanorods deposited on HOPG by AFM manipulation techniques and found a distinct anisotropy in nanoparticle movement depending whether the rod was moved parallel or perpendicular to its main axis. These observations could then be linked to the alignment between the organic ligands surrounding the nanorod and the substrate [56]. Polyakov et al. have used AFM manipulation techniques to analyzed the static and kinetic friction of $\mathrm{ZnO}$ nanowires [43]. In contrast to other approaches where friction is quantified by measuring cantilever torsion, the AFM tip is in this case employed only as a manipulation tool, while the friction was determined from the bending profiles of the nanowires. Some examples of different nanoparticles used for manipulation experiments in nanotribology are shown in Fig. 3.

Recent publications have shown that nanostructures suitable for manipulation experiments can also be gained from a variety of other inventive experimental strategies. For example, Bombis et al. have shown that
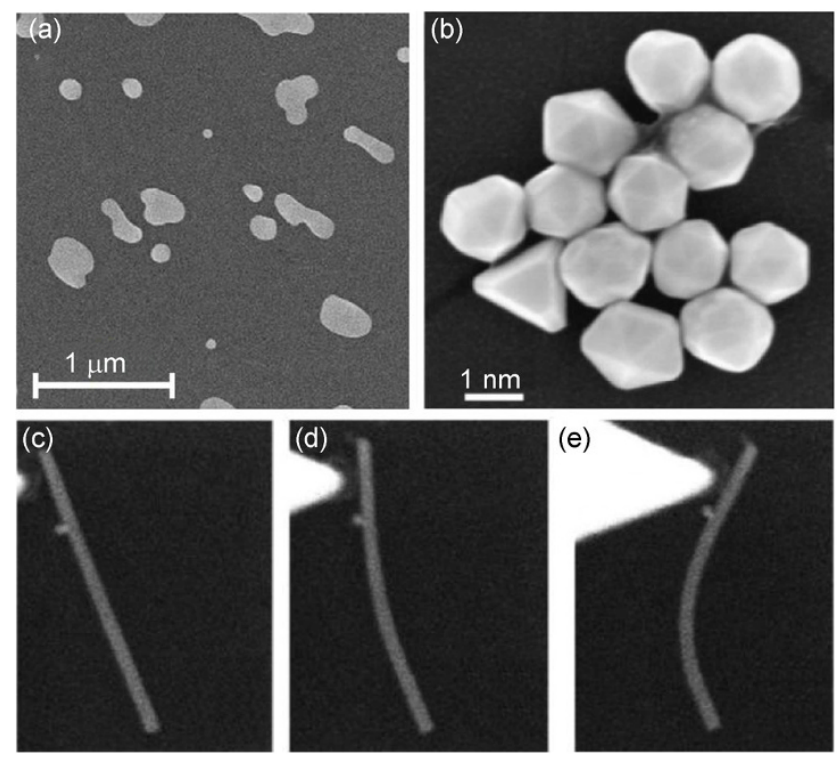

Fig. 3 Scanning electron microscopy images of different nanoparticles and nanostuctures used for tribological nanomanipulation experiments. (a) $\mathrm{Sb}$ nanoparticles prepared by thermal evaporation under UHV conditions onto HOPG. The distribution of different particles sizes illustrates how such particles can be utilized to analyze contact area-related questions (Reproduced with permission from Ref. [45], Copyright APS, 2008). (b) Colloidal gold nanoparticles from solution transferred onto a Si-substrate. While these particles all are of very similar size, their shape varies considerably, which makes them ideal objects to study effects like rolling vs. sliding of nanoparticles (Reproduced with permission from Ref. [50], Copyright Elsevier, 2012). (c)-(e) ZnO Nanowire of $150 \mathrm{~nm}$ diameter and $\approx 2.2 \mu \mathrm{m}$ length on a Si-wafer in interaction with an AFM-tip. The sequence of images shows how a continuously increasing bending of the wire is induced by the AFM tip (Reproduced with permission from Ref. [43], Copyright Beilstein Foundation, 2014). 
after evaporation of $\mathrm{NaCl}$ on $\mathrm{Cu}(111), \mathrm{NaCl}$ nanoparticles suitable for manipulation can be cut from larger $\mathrm{NaCl}$ structures [57]. This approach is especially interesting since size and shape of such nanoparticles can in principle directly be controlled. In contrast, Feng et al. have reported on the mobility of graphite flakes on graphene, where the flakes have been prepared by $\mathrm{H}_{2} \mathrm{O}$ assisted cracking of graphene sheets [58]. The results confirmed the strong dependence of interfacial friction on the relative orientation between flake and substrate, as previously reported by Dienwiebel et al. for a graphite flake trapped between AFM-tip and HOPG substrate [31].

\section{Experimental concepts: Measuring interfacial friction by nanoparticle manipulation}

The possibility to manoeuvre a sharp tip across a surface with sub-nanometer precision makes the AFM an ideal choice for any nanomanipulation task. As a consequence, the AFM has become a standard instrument to manipulate nanoscale objects in physics, engineering and biology. However, in nanotribology mere manipulation of nanoscale objects is not sufficient, as the interfacial friction force or the dissipated energy needs to be recorded during the manipulation process. In order to do so, several experimental strategies have been developed in recent years.

For conventional topography measurements, an AFM is typically operated in two main modes: In the contact mode, which is also referred to as static mode, tip and sample are in direct mechanical contact. This technique can be used to obtain nanometer resolution images on a wide variety of surfaces. Higher resolution, however, is often achieved using dynamic modes like the tapping mode [59] or the noncontact mode [60], where the cantilever oscillates in very close proximity to the sample surface. For both the contact and the dynamic mode, it has been shown that they can be successfully applied to facilitate nanoparticle sliding with simultaneous assessment of energy dissipation $[39,61]$. The experimental approaches to nanoparticle manipulation resulting from extending each of the two modes are described in the following subsections.

\subsection{Nanoparticle manipulation using dynamic operational modes}

Although there is a variety of different dynamic AFM modes that could in principle all be applied for nanomanipulation tasks, manipulation of nanoparticles is nowadays almost exclusively performed in tapping mode [59], where the cantilever is typically oscillated close to its resonance frequency while the oscillation amplitude serves as feedback parameter. The energy that is dissipated during one oscillation cycle $\Delta E$ is then a function of cantilever spring constant $c_{z}$, the quality factor $Q$, and the drive and oscillation amplitudes $a_{\mathrm{d}}$ and $A$, respectively [62]:

$$
\Delta E=\pi c_{z}\left(A a_{\mathrm{d}} \sin (\phi)-\frac{A^{2}}{Q} \frac{f_{\mathrm{d}}}{f_{0}}\right)
$$

Here, $f_{\mathrm{d}}$ and $f_{0}$ are the driving and oscillation frequencies and $\phi$ is the phase shift between them. In this mode, energy is transferred to the nanoparticle when the oscillating tip hits the rim of the nanoparticle (see Fig. 4), whereby the amount of energy transferred can be controlled by the driving parameters of the cantilever oscillation.

Based on this concept, Ritter et al. have demonstrated how the manipulation of latex spheres on HOPG can be controlled [61] by placing the cantilever at the edge of the particle (Fig. 4(a)). A small oscillation amplitude

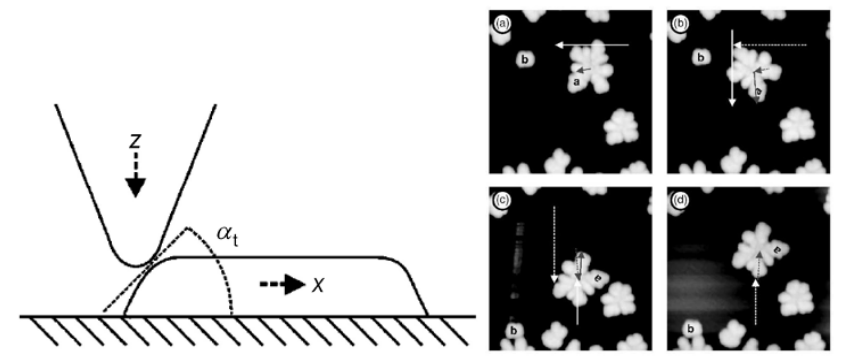

Fig. 4 Left: Sketch of the tip-particle coupling. The impact angle at between tip and antimony particle determines the normal $(z)$ and lateral $(x)$ components of the acting force [63]. Right: Illustration of the dynamic mode manipulation procedure for $\mathrm{Sb}$ on $\mathrm{HOPG}$ (image size $1 \times 1 \mu \mathrm{m}^{2}$ ). (a) Overview of the particle of interest (labeled with a) and the surrounding area. A white and a gray arrow indicate the path of the subsequent tip motion and the resulting dislocation of the particle, respectively. (b) Topography after the manipulation, showing a lateral translation of $83 \mathrm{~nm}$ and an in-plane rotation of $58^{\circ}$. (c) Result of the second manipulation step, and (d) final result after the third manipulation step (Reproduced with permission from Ref. [48], Copyright APS, 2005). 
was usually not sufficient to induce motion but instead allowed for imaging of the particle. Subsequently, the oscillation amplitude was increased until the threshold of particle movement (i.e., the friction force) was surpassed and the particle started moving. Fig. 4(b) $-4(d)$ illustrate the manipulation of antimony nanoparticles on HOPG.

Extracting reliable friction forces from such tapping mode manipulation experiments is, however, challenging. While theoretical analysis confirms that the measured energy dissipation is in fact a measure of the lateral forces occurring during manipulation [63], it is the unknown contact angle between the nanoparticle and the AFM tip (Fig. 4(a)) that limits the ability to accurately determine how much of the overall energy dissipation experienced by the cantilever is actually transferred into lateral motion. On the plus side, the dynamic mode is characterized by a very high degree of flexibility, as particle motion in arbitrary directions can be performed. Furthermore, the range of lateral forces that can be applied to the particle for translation is related to the square of the oscillation amplitude, which yields a very large dynamic range. The excitation amplitude can indeed be adjusted over orders of magnitude, which allows to easily switch between gentle imaging and manipulation of even very big particles. A detailed discussion of nanoparticle manipulation using dynamic modes can be found in Ref. [64].

The above discussion shows that very controlled manipulation of individual particles is possible. For its successful realization, however, it is necessary to have an electronic AFM control system available such as the one used to perform the particle manipulation shown in Fig. 4, where arbitrary tip motions along user-defined trajectories have been carried out under full feedback control. Most commercial AFM control electronics, however, do not support such procedure. To overcome this problem, a different approach relies on the statistical movement of a large ensemble of particles of similar size. Mougin et al. [44], Darwich et al. [65] and Paolicelli and co-workers [49, 66] have systematically analyzed the amplitude threshold necessary to induce particle motion in dynamic mode during continuous surface scanning. For colloidal gold nanoparticles this allowed to analyze how friction is influenced by particle-substrate chemistry, temperature, particle size and morphology. Similarly, Gnecco et al. report a detailed analysis of particle trajectories due to the impact between the oscillating tip and the particle within one scan frame [42].

Thus far, our considerations were all based on the application of the tapping mode, which is commonly used for nanoparticle manipulations. This mode is, however, not suitable for UHV conditions [60]. Instead, the frequency modulation (FM) mode, which is based on tracking changes in the cantilever's resonance frequency as it approaches the sample surface, is best employed for imaging in vacuum. But when it comes to particle manipulation, applicability of FM-AFM is limited due to its operational scheme that relies on self-excitation of the cantilever, which is very sensitive against perturbations. Therefore, trying to manipulate typical nanoparticles with contact areas larger than a few $100 \mathrm{~nm}^{2}$ most commonly results in the breakdown of cantilever oscillations, making the FM mode inapt for nanoparticle manipulations. Nonetheless, it has been shown how FM-AFM mode can be applied to move very small structures like single atoms [67] or perylene-3,4,9,10-tetracarboxylic dianhydride (PTCDA) molecules [68] with simultaneous assessment of the forces required to move the atoms or molecules.

\subsection{Nanoparticle manipulation using static mode atomic force microscopy}

\subsubsection{Pushing nanoparticles from the side}

As an alternative to nanoparticle manipulation performed in tapping mode, manipulation can also be carried out during contact mode operation of the AFM $[40,41,39,69,70]$. Best results are achieved if the AFM is operated in non-contact mode (either constant-amplitude mode [60] or constant-excitation mode [71, 72]) to record topography images without unwanted particle displacement prior to performing the actual nanoparticle manipulation by pushing the nanoparticle from the side in contact mode (Fig. 5) [73]. This mode is sometimes referred to as "Tip on Side" mode [73].

Figure 6 illustrates such a manipulation process with the example of an antimony particle on HOPG. From a topography image obtained in noncontact mode (Fig. 6(a)), the particle to be manipulated is chosen. The tip is subsequently positioned beside the 
(a)

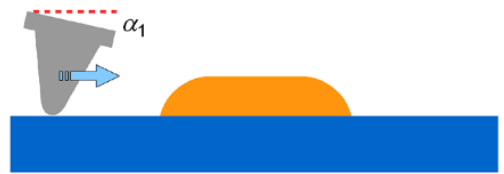

(b)

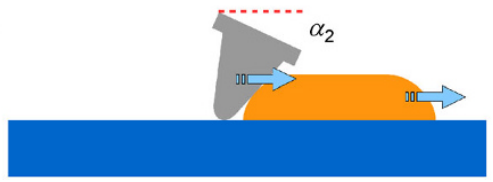

Fig. 5 Concept of contact mode manipulation of nanoparticles by pushing from the side ("Tip on Side" mode). (a) Before the tip reaches the nanoparticle, friction originates solely from the contract between the tip and the substrate resulting in a cantilever torsion $\alpha_{1}$. (b) Once the tip pushes the nanoparticle, both the tip/substrate and the tip/particle interface contribute to the overall friction. Thus, an increased torsion $\alpha_{2}>\alpha_{1}$ is measured, with the difference in torsion $\alpha_{2}-\alpha_{1}$ corresponding to the friction between nanoparticle and substrate.
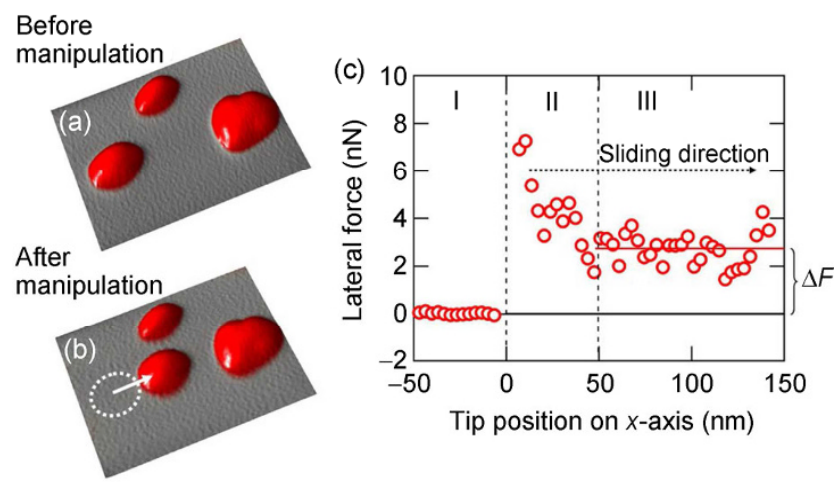

Fig. 6 Example of a particle manipulation based on contact mode manipulation ("Tip on Side" mode). (a) Topography image of a group of three $\mathrm{Sb}$ nanoparticles on HOPG before manipulation. (b) Topography image of the same group of nanoparticles after manipulation, where the middle particle has been pushed to the right. (c) Lateral force signal recorded during the manipulation process. The AFM tip slides on the bare substrate (regime I) before the onset (onset of manipulation can be observed in regime II). Finally, a constant particle translation is registered in regime III. The particle's kinetic frictional resistance (marked by $\Delta F$ ) can be determined as the difference between regime I and II (Reproduced with permission from Ref. [33], Copyright APS, 2013).

nanoparticle and the system is switched to contact mode. By moving the AFM tip along a straight pathway, the nanoparticle can now be displaced. The lateral force signal recorded during the manipulation process is shown in Fig. 6(c). Using the lateral force signal before the tip reaches the nanoparticle as reference to assess the friction between tip and substrate $(x<0$ in Fig. 6(c)), the additional interfacial friction between particle and substrate $\Delta F_{\mathrm{L}}$ can directly be determined from the difference between the two levels of the lateral force signal before and during the pushing of the nanoparticle (see Fig. 6(c)). Occasionally, peaks in the lateral force signal are observed directly after the tip gets into contact with the nanoparticle. Such peaks can either be due to the static friction of the nanoparticles or they might be related to surface defects, which may not be uncommon as such defects aid particle nucleation during sample preparation. After the manipulation process, the AFM is switched back to noncontact mode to record a control image and verify the successful particle displacement (Fig. 6(b)).

The cantilever normal force applied during this manipulation process is a crucial parameter since it determines whether the AFM tip will actually push the particle or if the tip rather traces the particle topography without any displacement taking place. A high normal force will typically result in particle manipulation, whereas low normal forces are more likely to enable accurate tracing of the particle topography. This dependence of the manipulation process on the normal force is utilized in an alternative manipulation scheme. After imaging the particles using a sufficiently low normal force, the AFM is operated in contact mode imaging with a normal force close to the threshold of manipulation. When the tip is now scanned across an area with a particle, it is probable that any particle will be pushed laterally after being imaged for a few scan lines [70, 45], resulting in a topography image where the particle seems to be "cut" (Fig. 7). The particle translation itself will result in a friction trace comparable to Fig. 6(c). The main advantage of this manipulation approach is that it allows to measure friction for a number of particles in a relatively short time because often several displacements can be observed during one image. But since there is no way to precisely control the tip-particle interaction, it is difficult to avoid unwanted nanoparticle motion. Thus, translation of a specific nanoparticle in a well-defined way is challenging. This drawback may, however, in many circumstances be out-weighed by the fact that particle manipulation experiments may be performed with even the most basic atomic force microscopes providing neither dynamic modes nor allow for arbitrary tip paths. Only if small, very mobile 


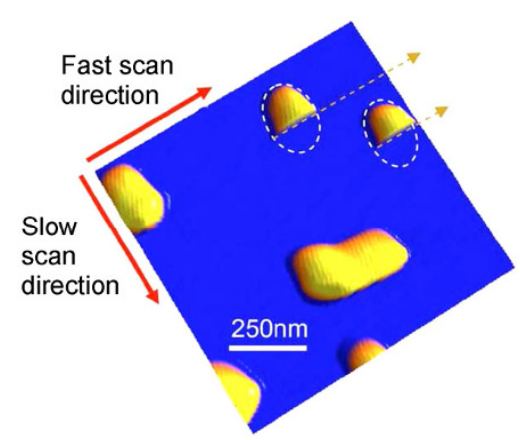

Fig. 7 Example of particle manipulation strategy relying on conventional $x y$ scans using a constant normal force slightly above the threshold for manipulation for small particles. The topographic image of $1 \times 1 \mu \mathrm{m}^{2}$ shows a group of particles. As indicated by the dashed lines, two particles are pushed to the right and out of the scan range after the particles have been correctly profiled for about half of their size, giving the particles the appearance of being cut in half (Reproduced with permission from Ref. [70], Copyright AIP, 2007).

nanoparticles are to be examined, using dynamic modes for imaging may become mandatory, since such particles can often not successfully be imaged using contact made.

\subsubsection{Nanoparticle trajectories during manipulation}

When pushing a nanoparticle from the side, both the tip and the nanoparticle should ideally move the same distance along a straight line. However, the shapes of tip and nanoparticle can lead to force components perpendicular to the tip path [42,49], which can cause the contact between tip and nanoparticle to break. Once this happens, the AFM tip will continue its path but leave the nanoparticle behind. During nanomanipulation experiments, this effect can be minimized by trying to direct the tip trajectory through the center of mass of the nanoparticle. It can nonetheless be problematic with respect to the accuracy of particle positioning and, more importantly, it can also affect the friction force measured during the particle manipulation. For the simplest case of a round particle and a round tip, the geometrical configuration used to calculate the particle trajectory is depicted in Fig. 8. For the calculation of the particle trajectories, it is assumed that any dependence of friction on sliding direction and sliding velocity can be neglected. Furthermore, the tip radius was assumed to be pointlike. The theoretical equations derived from this configuration [73] have subsequently been used to analyze the friction signal measured for an off-center manipulation of an antimony nanoparticle on HOPG (see Fig. 9). In this experiment an antimony nanoparticle of about $150 \mathrm{~nm}$ diameter was pushed from the side by the AFM tip and after manipulation, a considerable displacement perpendicular to the tip path was found (Figs. 9(a) and (b)). The corresponding lateral force signal shows the typical steep increase when the tip hits the nanoparticle, but starts to decrease

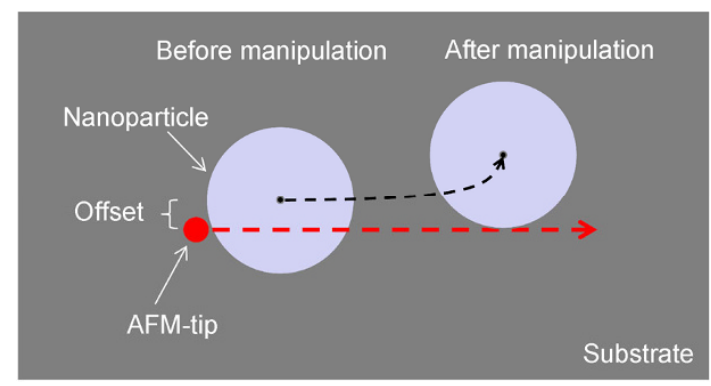

Fig. 8 Schematic of geometry during off-center manipulation. The position of the nanoparticle is shown in relation to the AFM tip and the tip path directly before and after the manipulation takes place. The dotted line indicates the particle trajectory during manipulation. The most crucial parameter to describe the manipulation process is the offset between the AFM tip and the center of mass of the nanoparticle measured perpendicular to the tip path.
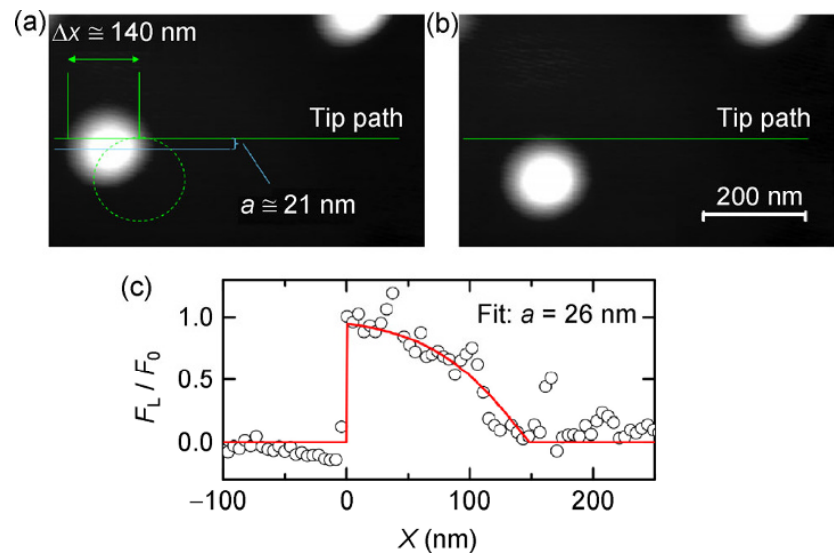

Fig. 9 Example of a manipulation event where the tip was moved from left to right and pushed an nanoparticle downwards during manipulation. (a) Topography image of the $\mathrm{Sb}$ nanoparticle on HOPG substrate before manipulation, (b) topography image after manipulation. The tip path and the position of the nanoparticle after manipulation are indicated in (a) and allow to estimate the offset $a$ to $\approx 21 \mathrm{~nm}$. (c) Lateral force signal measured during the manipulation. A fit to the experimental data yields $a=26 \mathrm{~nm}$ (Reproduced with permission from Ref. [73], Copyright Springer, 2010). 
immediately until the fricton is back to the initial level after approximately $150 \mathrm{~nm}$, meaning that the tip has lost contact with the nanoparticle. This behaviour can be well fitted by a theoretical friction profile calculated for an offset a of $26 \mathrm{~nm}$ between nanoparticle and tip. From the AFM images measured before and after nanoparticle manipulation, the offset a can be estimated to $21 \mathrm{~nm}$. Thus, fit parameter and directly measured offset are in good agreement. If, as it is the case in Fig. 9, the lateral force signal can be described by a theoretical model, the interfacial friction can still be precisely determined. However, such a calculations become increasingly complicated for more irregularly shaped particles and it is therefore preferably from a practical point of view to limit any quantitative analysis to particle manipulations with straight trajectories.

In other experiments by Gnecco and co-workers, the continuous off-center manipulation of nanoparticles during imaging has been employed to force groups of nanoparticles onto common resulting trajectories, an approach which can be used for arranging nanoparticles on surfaces or for sorting of nanoparticles. In order to do so, Rao et al. have scanned a Si surface covered with a number of round colloidal gold nanoparticles in tapping mode while the tip-sample interaction was chosen to be well above the threshold of manipulation [42]. This way, whenever the AFM tip hits a nanoparticle, the nanoparticle is displaced according to a theory similar to the one used to describe contact mode measurements [42, 74]. Again, the determining factor is the offset between the tip path and the nanoparticles center of mass. According to the findings of Rao et al., this parameter can be tuned by the line spacing during imaging with the effect that the nanoparticles can be forced onto straight trajectories whose effective angle relative to the scan direction can be varied by adjustig the line spacing (see Fig. 10).

Recently, Nita et al. have applied the manipulation concept developed by Rao et al. to push antimony nanoparticles of complex shape by contact mode AFM techniques on $\mathrm{MoS}_{2}$ [75]. In their experiments, however, the shape of the particles led to trajectories far more irregular than the ones shown in Fig. 10(a). Nonetheless, by using the precise particle shape as input for numerical simulations, the particle trajectory
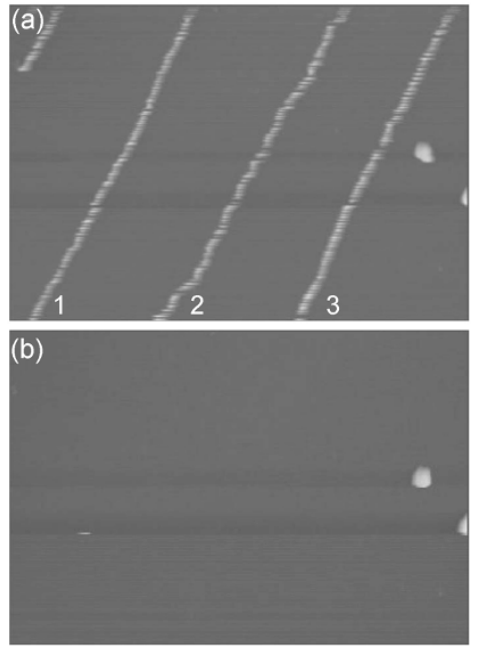

Fig. 10 Topography images recorded in tapping mode during forward scan (a) and backward scan (b). The forward scan (a) shows three parallel trajectories of gold nanospheres on a $\mathrm{Si}$ substrate. No trajectories are found in the backward scan (b), which means that the particles are efficiently pushed out of the tip's path during the forward scan and are therefore not interacting with the tip during the backward scan (Reproduced with permission from Ref. [74], Copyright APS, 2009).

could accurately be described and quantitative data for the interfacial friction was extracted [75]. We therefore conclude that despite the need to apply non-trivial theoretical models to extract quantitative data, the results obtained in contact mode by various groups suggest that the approach to study friction through nanoparticles manipulations occurring during standard AFM operation (i.e., "imaging" with slightly higher maximum normal loads) still represents a promising route for future investigations as all necessary data can be gathered with a very simple procedure.

\subsection{3 "Tip-on-Top" approach}

A slightly different approach for particle manipulation is realized by placing the tip on top of the particle during manipulation instead of placing it at the side. In this approach, which is illustrated in Fig. 11, the tip is first positioned approximately in the center of the nanoparticle's top surface. If then tip motion is initiated, two scenarios are possible: (1) The tip slides over the surface of the nanoparticle with the lateral force signal reflecting the friction between tip and nanoparticle, or (2) the nanoparticle is following the tip motion by gliding over the substrate. In this case, which we will refer to as the "tip-on-top" manipulation mode, the measured torsional signal is directly 
(a)

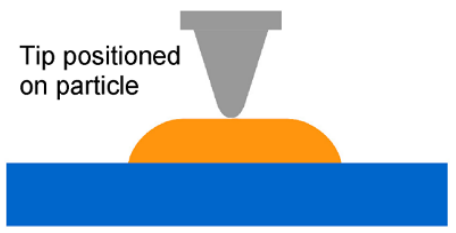

(b)

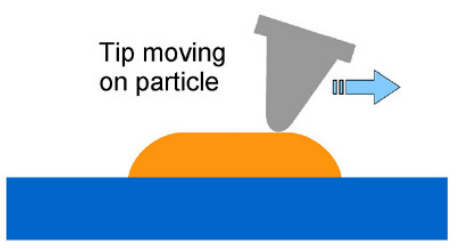

(c)

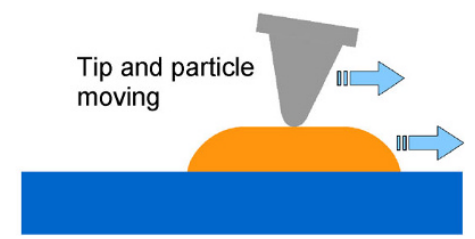

Fig. 11 Figure illustrating nanoparticle manipulation by employing the "tip-on-top" approach described in the text. (a) The tip is positioned on top of a particle (starting position). (b) If motion of the cantilever is initiated and the cantilever normal force is below a certain threshold value, the cantilever is sliding on the particle, profiling the nanoparticle's top surface. (c) If the cantilever load is above the threshold, the tip remains on a fixed position on top of the particle and tip and particle will move together.

proportional to the interfacial friction between particle and substrate.

The crucial parameter that distinguishes between the two scenarios is the ratio of the shear forces in the tip-particle-substrate system. Only if the lateral force needed to shear the tip-particle interface is larger than the force required to shear the particle-substrate interface, the particle moves together with the tip. If this is the case nanoparticles can be moved over large distances while the tip is placed on top of them. An example is given in Fig. 12, where an antimony particle has been displaced under UHV conditions in the "tip-on-top" mode.

One strategy to perform nanoparticle manipulations using the "tip on top" mode is to first operate the AFM in noncontact mode and placing the tip either on the left or right hand side of the chosen nanoparticle (Fig. 12(a), position marked by the cross). Then the tip is first scanned across half of the particle (as indicated in Fig. 12(a)), placing it directly on top of the nanoparticle (Figs. 12(a) and 12(c) for $x=0$ ). At this position the AFM is switched in situ from noncontact to contact mode [70,73] and the cantilever normal force is slowly increased, allowing to exert a sufficient lateral
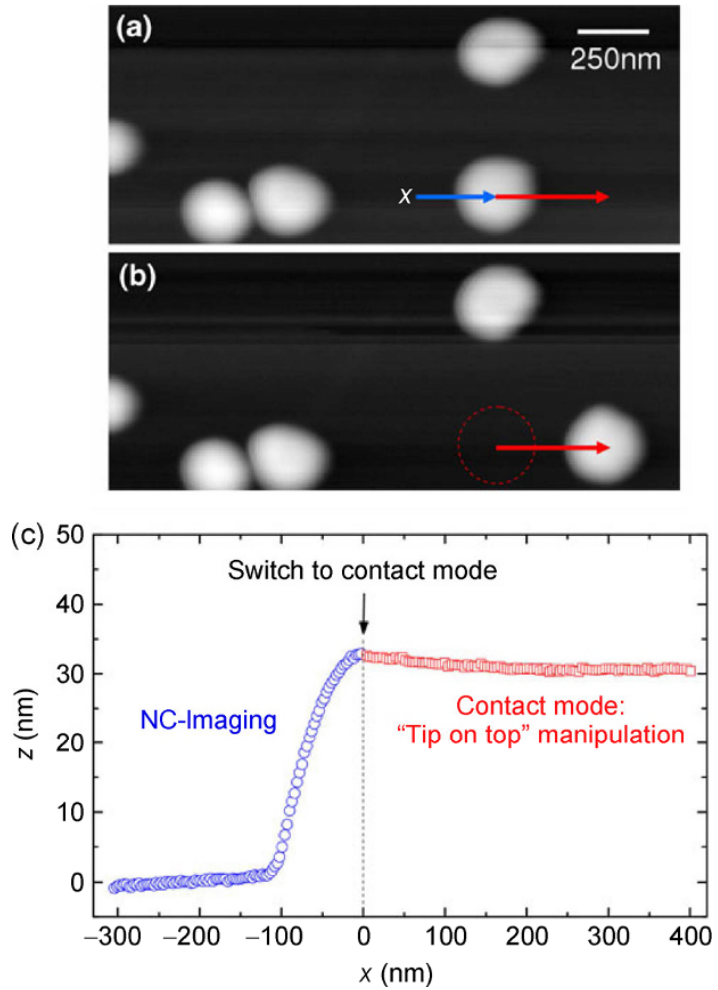

Fig. 12 Example of a controlled manipulation performed in the "tip-on-top" mode. (a) Noncontact topography image before manipulation. The cross indicates the initial cantilever position, whereas the two arrows mark the paths used to position the cantilever on top of the particle and to perform the manipulation. (b) Noncontact topography image after the nanoparticle manipulation along the vector path. (c) Topography signal measured during the two vector pathways. The tip is first positioned on top of the nanoparticle in noncontact mode $(x \leqslant 0)$; the subsequent manipulation is the carried out in contact mode $(x \geqslant 0)$ (Reproduced with permission from Ref. [73], Copyright Springer, 2010).

force for moving the particle. Once the normal load has been set, the tip is moved along a second vector (indicated in Fig. 12(a)) to perform the controlled nanoparticle movement. During the manipulation the topography signal and the lateral force signal are recorded. Given a flat substrate surface the topography signal remains flat over the whole pathway of the manipulation (Fig. 12(c), $x \geqslant 0$ ) as long as the tip remains firmly on top of the nanoparticle. After the particle movement is completed, the AFM is switched back from contact to noncontact mode and a control image is recorded (Fig. 12 (b)) verifying the nanoparticle's manipulation path. If the normal load is sufficient, we find the nanoparticles to firmly follow the tip movements, allowing controlled manipulations with over $1 \mu \mathrm{m}$ of displacement [51]. 
If quantitative values for interfacial friction are to be extracted from "tip-on-top" manipulations, one has to keep in mind that in this case a single onedirectional nanoparticle manipulation lacks an absolute reference level necessary to quantify the interfacial friction. Therefore, quantitative friction data must be extracted from the forward and backward motion of the nanoparticle, a procedure similar to recording friction loops in conventional friction force microscopy (see Section 2.3).

\subsection{Identifying static friction in nanoparticle manipulation experiments}

One important aspect in attaining a coherent picture of friction processes at the nanoscale is the correlation between static and sliding friction. While this difference is a well-known fact for friction experiments on the macroscale, it is less clear if or how this concept is applicable to nano- or mesoscale contacts, where stick-slip motion is considered to be the dominant process. Currently, interest is especially spurred by new concepts that take ageing of nanocontacts into account [23, 76-78]. In many cases, nanotribological ageing effects can principally be analyzed by velocitydependent measurements [20, 23], since the stick phase during stick-slip motion can be considered as a hold time during which contact ageing can occur. However, velocity-dependent measurements only allow to vary the hold times in a certain range. To achieve longer hold times with saturated contact ageing, it might be required to suspend the sliding motion alltogether and reinitiate it after the desired amount of time.

Again, the well-defined interfaces between nanoparticles and substrates can form ideal model systems to undertand nanoscale processes related to static friction. To measure the static friction of nanoparticles, different strategies have successfully been employed. One of the first examples was presented by Luethi et al., where the $\mathrm{C}_{60}$ nanoparticle on $\mathrm{MoS}_{2}$ was pushed from the side and the torsion of the cantilever interacting with the nanoparticle was used as a measure of interfacial friction. In this case, similar to Fig. 6, the static friction resulted in a high cantilever torsion that was built up right before the nanoparticle started moving [39]. Once the particle was sliding steadily, a reduced lateral force signal was measured. However, the steep increase of the lateral force signal when the cantilever hits the particle can make it difficult to identify the exact maximum, which can be interpreted as static friction, especially if typical point densities for data acquisition are used. Moreover, the process of contact formation between the tip and the nanoparticle can influence the measurement, resulting in unreliable information about static friction.

Tripathi et al. have used tapping mode manipulation techniques to assess the temperature dependence of static friction for gold nanoclusters on HOPG [66]. By measuring the threshold of amplitude reduction at which detachment occurs, they could quantify static friction and found that the detachment of small gold nanoclusters with diameters of about $27 \mathrm{~nm}$ can be described as a thermally activated process, resulting in lower detachment energies measured at higher temperatures [66].

Another possible approach to distinguish between static and sliding friction is based on the "tip on top" manipulation scheme [51]. With the tip resting on top of the nanoparticles, there are two possible modes of tip and cantilever movement (see Fig. 11): The tip can either move on top of the nanoparticles, in which case the lateral force signal represents the friction between tip and particle, or the contact between tip and particle is firm, in which case the tip drags the particle along and the lateral force signal represents the friction between particle and substrate. The key parameter to control the sliding behavior is the cantilever normal force, which can be used to facilitate the transition between static and sliding friction. The principle scheme is depicted in Fig. 13 for an $\mathrm{Sb}$ nanoparticle on HOPG: First the tip is positioned on top of the nanoparticle and a contact mode scan of a small area (typically $A=20 \mathrm{~nm}^{2}$ ) in the center of the particle is initiated. This scan starts at a low cantilever load, in which case the lateral force between tip and particle is not sufficient to overcome the static friction between particle and substrate. By gradually increasing the cantilever load with time $t$, the friction between tip and nanoparticle will increase (Fig. 13(c)) until the lateral force is sufficient to overcome the static friction of the nanoparticles $(t=1.85$ in Fig. 13(c); note that $t$ is measured in arbitrary units). The nanoparticle is now moving together with the tip and the 
(a)

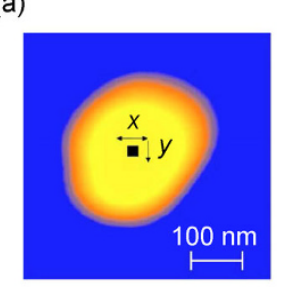

(c)

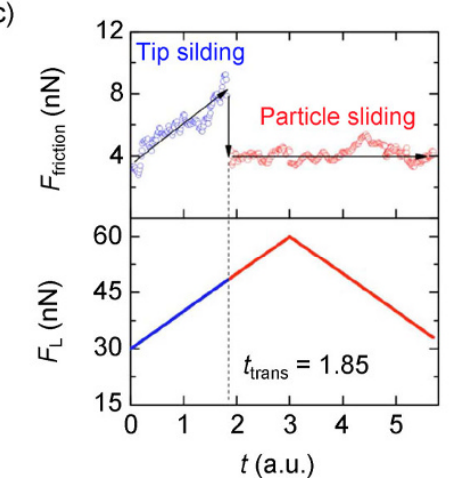

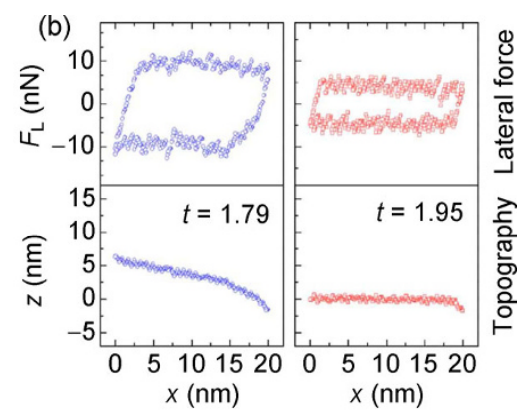

Fig. 13 Distigushing static and sliding friction using the "tip on top" approach. (a) Sb nanoparticle ob HOPG substrate, where the typical scan area is indicated. (b) Friction loops (top) and topography (bottom) just before (left hand panels) and after (right hand panels) particle sliding was initiated. (c) Plots of the effective friction force $F_{\text {friction }}$ (determined from friction loops such as the ones in panel (b) and cantilever normal force $F_{\mathrm{N}}$ as a function of time $t$ while continuously scanning the AFM tip on top of the nanoparticle $\left(A_{\text {contact }}=68,000 \mathrm{~nm}^{2}\right)$ with a scan range of $20 \mathrm{~nm} \times 20 \mathrm{~nm}$. The sudden drop at $t_{\text {trans }}$ indicates the transition to particle sliding (Reproduced with permission from Ref. [51], Copyright AIP, 2009).

friction level in Fig. 13(c) (red part) represents the sliding friction of the nanoparticles, whereas the maximum frictional force reached at $t=1.85$ (blue curve) can be interpreted as static friction of the nanoparticles. From Fig. 13(c) it can be seen that in case of the moving nanoparticles no further load dependence can be observed, which is indicative that the adhesion between nanoparticle and substrate is much larger than any applied cantilever load. The transition of dynamic states becomes also obvious from Fig. 13(b). Right before the transition, the friction loop is fairly wide, while the related topography signal has a considerable slope related to the shape of the particle, which is not flat. After the transition, however, the friction loop is not only significantly narrower, but also the slope in topography has vanished since the topography signal is now related to the nanoparticle sliding on the flat HOPG substrate.

In the same work, it was also shown that the transition from static to sliding friction is reproducible, meaning that the transition from static to sliding friciton can be repeated several times for the same nanoparticles. Interestingly, for the limited number of particles of the same size investigated in Ref. [51], the ratio between static and sliding friction seemed to be constant with a typical ratio of $F_{\text {sliding }} / F_{\text {static }} \approx 0.5$.

So far, the exact reason for the observed difference between static and sliding friciton remains unclear. Due to the reproducibility of effects for the same nanoparticle, any wear-related interface changes can be ruled out. However, theory predicts that the behavior of a layer of mobile molecules trapped between the moving surfaces can dominate the phenomenon of static and kinetic friction due to a shear force-induced transition from a solidlike to a liquidlike structure of the interface layer [79]. In good agreement with the experimental result, Persson found that the ratio between kinetic and static friction is consistently one half for a wide variety of simulation parameters [79]. The model also predicts that if the lateral force is reduced again, the liquidlike state prevails until much below the initial threshold, giving rise to a hysteretic behavior as observed experimentally [51]. Nonetheless, in our UHV experiments it is difficult to imagine the presence of a layer of additional interface molecules. Still, it is astonishing that the model from Persson is very consistent with the experimental observations, including a kinetic/static ratio of one half and hysteretic behavior. This suggests that if no contamination particles are present, the last layer of $\mathrm{Sb}$ atoms in contact with the HOPG substrate might act as a de facto boundary lubrication layer.

\subsection{Comparison of manipulation strategies}

The various manipulation schemes introduced above have different advantages and drawbacks. In manipulations based on dynamic AFM modes, a high range of forces can be applied to the particles by simply adjusting the oscillation amplitude and arbitrary translation paths for the particles can be chosen. On the downside, a direct measurement of frictional force is not possible; instead, the momentum transfer to the particle is quantified through monitoring the system's energy dissipation during manipulation. 
Here, it often remains unclear how much of this energy is actually transferred into the nanoparticle motion.

In contact mode manipulation, on the other hand, the fixed lateral spring constant of the specific cantilever used significantly limits the range of frictional forces that can be accurately detected. However, the restriction might be outweighed by the ability to measure the frictional force between particle and substrate directly, allowing a straightforward quantitative analysis of interfacial friction. The manipulation pathways are more limited, since friction can only be measured perpendicular to the cantilever, but in principle static and dynamic friction can be distinguished. When placing the tip on top of the particles the manipulation control is even better, since a fixed contact between nanoparticle and tip exists. In this configuration, it is possible to perform multiple nanoparticle manipulations without braking the contact between tip and sample. Initial experiments showed that up to 100 consecutive manipulations are possible. This opens the door for measurements regarding two fundamental key parameters in nanotribology, namely load and velocity. For load dependent measurements, the cantilever can be used to exert a varying normal load on the particle during sliding. This enables in principle the measurement of the true load dependence of friction, an issue of considerable fundamental interest. In practice, however, this may be difficult to quantify as the additional load exerted by the cantilever may often be dwarfed by the intrinsic particle-substrate interaction due to adhesion (cf. Fig. 13). Furthermore, the "tipon-top" mode allows to vary the sliding velocity of the nanoparticle within an uninterrupted series of nanoparticle manipulations. Such experiments have recently been performed to clarify how the basic model of thermal activation can be transferred from small contact areas of AFM tips to the extended contacts of nanoparticles [52].

\section{Friction of extended nanocontacts: Contact area dependence and structural lubricity}

\subsection{Basic concepts of friction for extended nano- contacts}

Since the advent of friction force microscopy in 1987, most research in the field of nanotribology has concentrated on the analysis of very small contact areas, so-called nano-asperities. The interest to focus on nano-asperities was spurred by a suggestion made by Bowden and Tabor in the 1950's that arbitrarily rough surfaces can favorably be approximated by an ensemble of multiple asperities [9]. Since the contact between an AFM tip and a surface represents the ideal experimental realization for such single asperities, friction force microscopy allows to analyze the basic friction processes occurring at point contacts on the atomic scale. And indeed, thanks to focussing the experiments on very small contact areas, results could often be explained by theoretical models that reduced the contact to only a few atoms or even a single one $[17,23,20]$.

Considerably less research has, however, been done on extendend nanocontacts, even though they represent frequent constituents of realistic surfaces. As a consequence, many aspects of the frictional behavior of extended nanocontacts are still insufficiently explored. One of the most fundamental but unresolved questions in nanotribology concerns the question of how the frictional force $F_{\text {friction }}$ experienced at a finite, atomically flat interface of nanoscopic dimensions scales with the actual contact area $A_{\text {contact. }}$. The answer might even affect our understanding of the widely accepted classical friction laws of Amontons, who stated that friction is proportional to the normal force, but independent of the apparant contact area:

$$
F_{\text {friction }}=\mu F_{\text {load }}
$$

Here $F_{\text {load }}$ represents the external loading force and $\mu$ the friction coefficient, which depends only on the actual combination of materials in contact. Only under the assumption of a linear dependence between true contact area and friction, this law can be understood by the commonly acknowledged model first introduced by Greenwood and Williamson [80], where a linear dependence between the real contact area between two surfaces and the applied load on the interface is assumed.

However, not only the unresolved questions regarding contact area dependence of friction make extended nanocontacts an intriguing problem. Another aspect of fundamental interest is the fact that in contrast to atomic point contacts where mostly the 
interaction of single atoms is considered, the collective behaviour of a multitude of atoms within the two surfaces sliding relative to each other can be of significant importance. The importance of such collective behaviour becomes most obvious in the case of an effect called "structural lubricity", which, as will be described below in detail, depends on the degree of interlocking between the atoms of two extended surfaces.

One key parameter determining the interfacial friction between two sliders is the ratio of lattice constants. This can be rationalized by considering a simple scenario [10] as it is depicted in Fig. 14 with a number of substrate atoms and their corresponding periodic surface potential with lattice constant $a$. A single atom on such a surface will drop into one of the potential minima of the surface energy landscape (Fig. 14I). If, however, a cluster of two atoms would be put on the surface the resulting energy barrier per atom will strongly depend on their lattice constant $b$. If $a=b$ both atoms will drop to the same energy level as the single atom previously. However, $a \neq b$ will result in a considerable reduction of the energy barrier per atom for the cluster (Fig. 14II). This behavior continues with increasing cluster size (Fig. 14III) and ultimately leads to a vanishing energy barrier per atom as the particle size increases towards infinity. More detailed calculations show that this results in a sublinear increase of friction as a function of the contact area for any incommensurate, i.e., non-matching, crystalline interface $[32,81]$ (please note that although the energy barrier per atom vanishes if the particle size approaches infinity, this effect is outweighed for

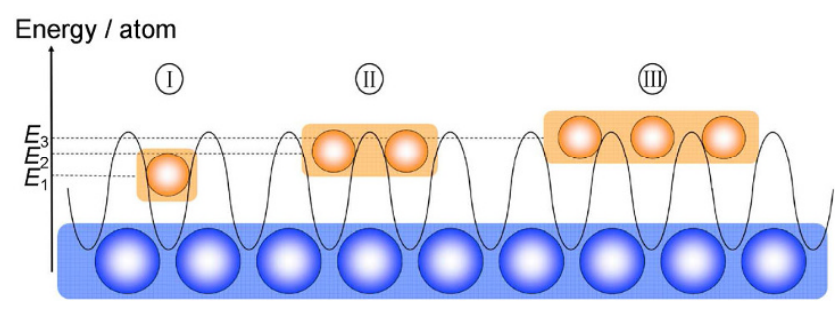

Fig. 14 Figure illustrating the effect of incommensurability on the average barrier between potential minima: While the number of atoms that have to overcome a barrier increases from one to three for (I) to (III), the height of the individual barriers shrinks significantly $\left(E_{1}>E_{2}>E_{3}\right)$. For increasingly larger contacts, the effective overall barrier height will approach zero even though a large number of atoms contribute to the frictional resistance. finite contact areas by the overall increase of particle atoms, which results in low but still finite friction).

A similar behaviour is anticipated for interfaces, where one or both surfaces are amorphous. In this case some atoms will go up the potential energy ramp while at the same time others go down, resulting in cancellation due to the averaging effect of probability theory's central limit theorem [82]. Assuming that the contact area $A_{\text {contact }}$ between substrate and slider is proportional to the number of atoms $N$ of the slider, it can be shown that interfacial friction between dry, amorphous and flat surfaces scales as $F_{\text {friction }} \propto \sqrt{A_{\text {contact }}}$, which at the same time means that friction per atom vanishes with increasing contact size, as it is the case for incommensurate lattices. The effect of low friction due to non matching interfaces has originally been termed "superlubricity" [83, 84]; however, as it is a purely structural effect and to distinguish it from other effects that may lower the interfacial friction, it has been suggested by Müser to denote it more adequately as "structural lubricity"[85].

Curently, many aspects of structural lubricity are still under debate, such as the exact circumstances under which a superlubric state can actually be established. Nonetheless, a growing number of experimental studies already seems to corroborate its existence [31, 45, 58, 86-89]. In this context, we note that the sublinear contact area dependence of friction predicted for this case is a unique key feature of structural lubricity and thus allows for an unambiguous identification of the underlying physical principle.

When analyzing the friction of nanoparticles, we may start with reflecting on whether or not a model that assumes the contact area of the nanoparticle to be completely rigid is sufficient to correctly predict the particle's overall frictional behaviour, since elastic deformations might cause the particle's actual behaviour to diverge significantly from the one derived from more simplistic rigid models. For example, Reguzzoni et al. have used molecular dynamics (MD) simulations to describe the onset of a slip process in a Xe film on a Cu substrate [90], a system where a commensurate contact can be assumed. If an external load is applied to this system, this can result in a frictional slip that originates from the nucleation of a small commensurate domain. By these simulations, Reguzzoni et al. could 
understand results obtained by Coffey at al. using a quartz crystal microbalance, where particle movement was observed in spite of an energy barrier that was too high to be overcome in the absence of the nucleation of structurally distinct domains [38]. More generally, even though elastic deformations of surfaces are thought to alter the behaviour of structural lubricity, calculations suggest that realistic deformations are too small to invalidate the basic conclusions drawn from rigid structure models [83, 91-93]. The remainder of this review will be devoted to an experimental analysis of this problem, which is complemented by a comparison with theory.

\subsection{Contact area dependence of friction analyzed by nanoparticle manipulation}

The above discussion highlights the importance to analyze the contact area dependence of friction for nanoscale systems. These questions are ideally addressed using nanoparticle manipulation, since particles of varying size can readily be produced in a well-defined manner (e.g., Fig. 3(a)) and allow to systematically study the contact area dependence of friction. However, atomically flat incommensurate interfaces do even under clean and well-ordered interface conditions not necessarily display structural lubricity. The reason for that may be the nature of interaction across the interface. If, e.g., strong chemical bonds between slider and substrate are possible, the interfacial friction should depend mainly on the number of bonds. Szlufarska et al. have used MD simulations to analyse this scenario for amorphous carbon tips on diamond substrates (both terminated with hydrogen) [94] and could determine for all $\mathrm{Si}$ atoms at the interface whether a bond to the substrate was formed or not, which was dependent on the proximity between $\mathrm{Si}$ atoms and the substrate. In this case, the contact area is defined by the region in which bonds are built and the number of bonds determines the friction and it scales proportional to this contact area with no reduction of friction related to structural lubrication effects.

\subsubsection{Frictional duality observed during nanoparticle sliding}

Two recent studies $[48,45]$, which for the first time used nanoparticle manipulation to analyze the contact area dependence of friction for extended nanocontacts, relied on antimony nanoparticles prepared by thermal evaporation on freshly cleaved HOPG (see Fig. 3(a)). In this case, no chemical bonds will be formed between substrate and particles, as attractive forces at the interface are restricted to van der Waals interaction. Based on results from an independent study [53], we also know that the compact shape exhibited by most of the particles is indicative of them being amorphous. As a consequence, the particle/substrate interface is expected to exhibit suberlubric behaviour. And even if some of the particles were crystalline, the atomic lattices of $\mathrm{Sb}$ and HOPG do not match, leading to incommensurate particle/substrate interfaces under all circumstances and eventually to the occurrence of structural lubricity. Experiments were performed both under ambient conditions $[48,45]$ and under UHV conditions [45] using either tapping mode manipulation [48] or contact mode manipulation [45]. Particle sizes varied from $10,000 \mathrm{~nm}^{2}$ to $300,000 \mathrm{~nm}^{2}$, illustrating the range of contact areas accessible to nanomanipulation experiments.

The measurements performed under UHV conditions resulted in two distinct friction branches (Fig. 15(a)), with the majority of particles showing a linear dependence of interfacial friction as a function of contact
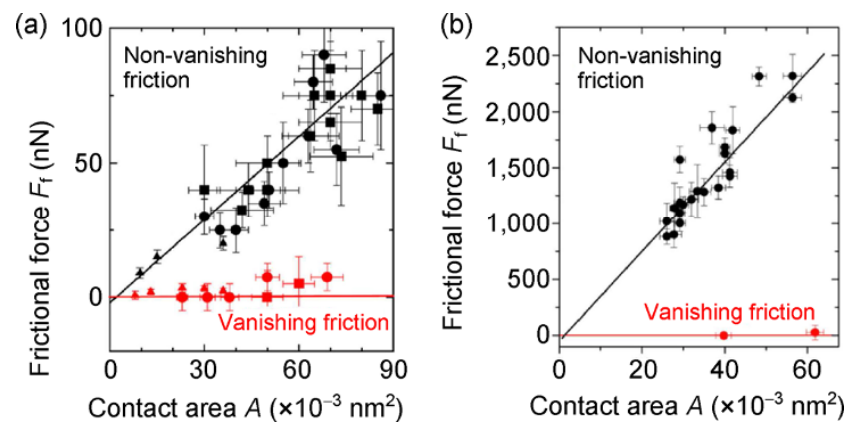

Fig. 15 Friction measured for antimony nanoparticles manipulated on HOPG under UHV conditions (a) and ambient conditions (b). The measurements performed under UHV conditions reveal two distinct regimes. While the majority of particles shows finite friction values, which can be described by a linear friction vs. area relation (black symbols), a considerable number of particles shows vanishing friction (red symbols) close to the limit of experimental sensitivity. This apparent frictional duality cannot be observed for the measurements under ambient conditions (b), where the data points are almost exclusively appendant to a linear branch, with only two data points showing vanishing friction (Reproduced with permission from Ref. [45], Copyright APS, 2008). 
area (black symbols). From these measurements, a shear stress of $\tau=F_{\text {friction }} / A=(1.04 \pm 0.06) \mathrm{MPa}$ was calculated. At the same time, a smaller fraction of nanoparticles was found that showed considerably lower friction, which was almost unquantifiable by the instruments sensitivity [45]. This apparent duality of friction among the nanoparticles, which have been prepared under UHV conditions and were transferred to the UHV-AFM directly afterwards without breaking the vacuum, was not equally pronounced for the measurements performed under ambient conditions. Both sets of experiments $[48,45]$ revealed a linear increase of friction with contact area but only two particles showed significantly lower friction during the contact mode experiments under ambient conditions (Fig. 15(b)).

This behaviour is in stark contrast to expectations based on the previously discussed characteristics of structural lubricity. Even though metallic nanoparticles evaporated on atomically flat HOPG should be ideal model systems for structural lubricity, the characteristic sublinear friction vs. area behaviour could not be observed and the majority of particles showed friction values that are incompatible with the low friction values expected for incommensurate interfaces. But while the results might seem surprising at first glance, the occurrence of high friction particles and the duality of friction can be rationalized by a theoretical model developed by $\mathrm{He}$ et al. [95], where mobile molecules were incorporated into an otherwise clean and ordered incommensurate interface (Fig. 16(b)). Corroborated by MD simulations, Müser et al. predicted that already small amounts of molecules "contaminating" the interface can prevent it from displaying structural lubricity [82]. In this scenario, the mobile molecules present in the interface act as mediators that induce an altered form of interlocking between the particle and the substrate (see Fig. 16(c)). Müeser et al. also predicted interface contamination to result in a constant shear stress $\mu$ independent of the contact area between particle and substrate [82], an effect that only weakly depends on the exact amount of interface contamination [82,95].

Based on this theory, the experimental results described above can be understood assuming that even under "clean" UHV conditions [45], a fair number of such mobile adsorbates can accumulate on HOPG (a)

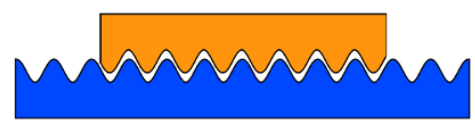

(b)

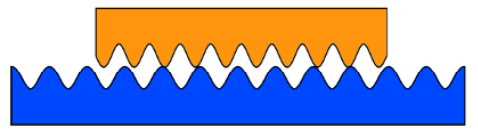

(c)

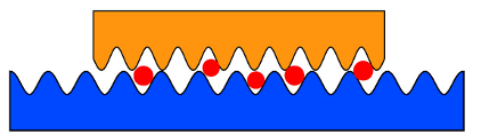

Fig. 16 Figure illustrating the effect of contamination on friction. (a) The atomic structure of two commensurate surfaces in contact can interlock, resulting in an area-independent friction coefficient. (b) Structural lubricity: Two incommensurate, atomically flat surfaces. The barrier between stable potential minima, and thus the friction per unit area, decreases with increasing contact size. (c) If the contact in (b) is contaminated by mobile molecules, the friction per unit area is again independent of the contact size. In this case, the mobile molecules can always lock at suitable potential minima, acting as molecular mediators between the incommensurate structures.

surfaces especially over extended measurement times. In this case, the results suggest that the interfaces of some particles are atomically clean while others experience a break-down of superlubricity due to contamination, which would elegantly explain the observed duality of nanoparticle friction [45] (Fig. 15(a)). On the other hand, contamination will undoubtedly have a much stronger impact for measurements performed under ambient conditions, which rationalizes the almost non-existence of the low friction branch (Fig. 15(b)).

Close observation of the island structure by AFM imaging shows no systematic correlation of particle features (e.g., morphology, degree of ramification, structure, height or orientation) with the occurrence of vanishing friction [46]. In fact, islands of comparable size and shape can show completely different frictional behavior within the same scan frame. Also, the friction measurements show a high degree of reproducibility during multiple translation of the same particle [70]. This suggests that indeed the properties of the particlesurface interface are decisive for the observed duality. Alternatively, an array of possible artefacts has been considered as possible origins for the occurrence of vanishing friction, but could eventually be dismissed (see Ref. [45] for details). 
3.2.2 The role of interface contaminations: Theoretical calculations

Although the universal presence of interface contamination seems to be a very plausible explanation for the observed duality in friction of nanoparticles, the question remains if conceivable concentrations of likely contaminants are really sufficient to quantitatively explain the friction levels observed in nanomanipulation experiments. In order to clarify this question, Brndiar et al. used density functional (DFT) modeling and analyzed the behaviour of different contamination atoms or molecules at the interface between HOPG and crystalline antimony [47]. By calculating the potential energy surface (PES) for the sliding process with and without contaminants, information could be gained about the additional energy barriers that need to be overcome in the presence of contaminants. Figure 17 illustrates the results for the case of a clean interface and an interface with an $\mathrm{H}_{2} \mathrm{O}$ molecule trapped between the surfaces of $2.6 \mathrm{~nm}^{2}$ size. While the clean interface results in a very low energy barrier $\left(E_{\text {barrier }}<1 \mathrm{meV}\right)$ that is consistent with the expectations for structural lubricity, adding an $\mathrm{H}_{2} \mathrm{O}$ molecule into the interface results in a substantial increase of the energy barrier, which is found to be approximately $100 \mathrm{meV}$. Similar effects and energy barriers were found when adding $\mathrm{Sb}_{4}$ clusters, oxygen atoms or $\mathrm{Sb}_{4} \mathrm{O}_{6}$ into the interface.

In more detail, the simulations by Brndiar et al. revealed that the trapped $\mathrm{H}_{2} \mathrm{O}$ molecule is moving along with the antimony surface, but still remains essentially mobile. This mobility is indicated in Fig. 17(b), where the rotation of $\mathrm{H}_{2} \mathrm{O}$ molecule during translation is plotted. Particle mobility is an important condition for contaminating "dirt molecules" to function as mediators between incommensurate interfaces [82]. If dirt molecules would be fixed and rigidly bound to, e.g., the particle, the resulting new interface would again show superlubricity.

In an attempt to estimate the number of contamination molecules at the interfaces, it was found, that approximately $1 \mathrm{H}_{2} \mathrm{O}$ molecule $/ 75 \mathrm{~nm}^{2}$ is required to explain the experimentally observed friction levels. This value seems to be conceivable, especially when

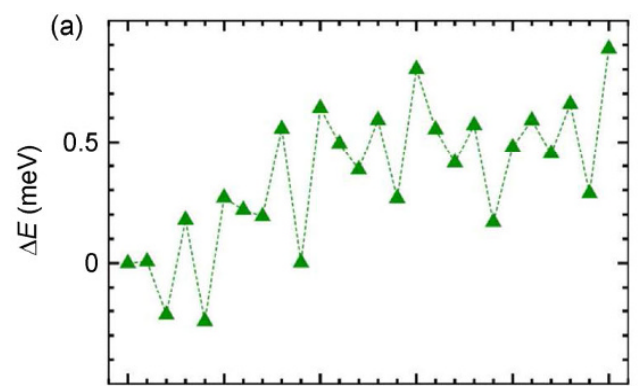

(c)
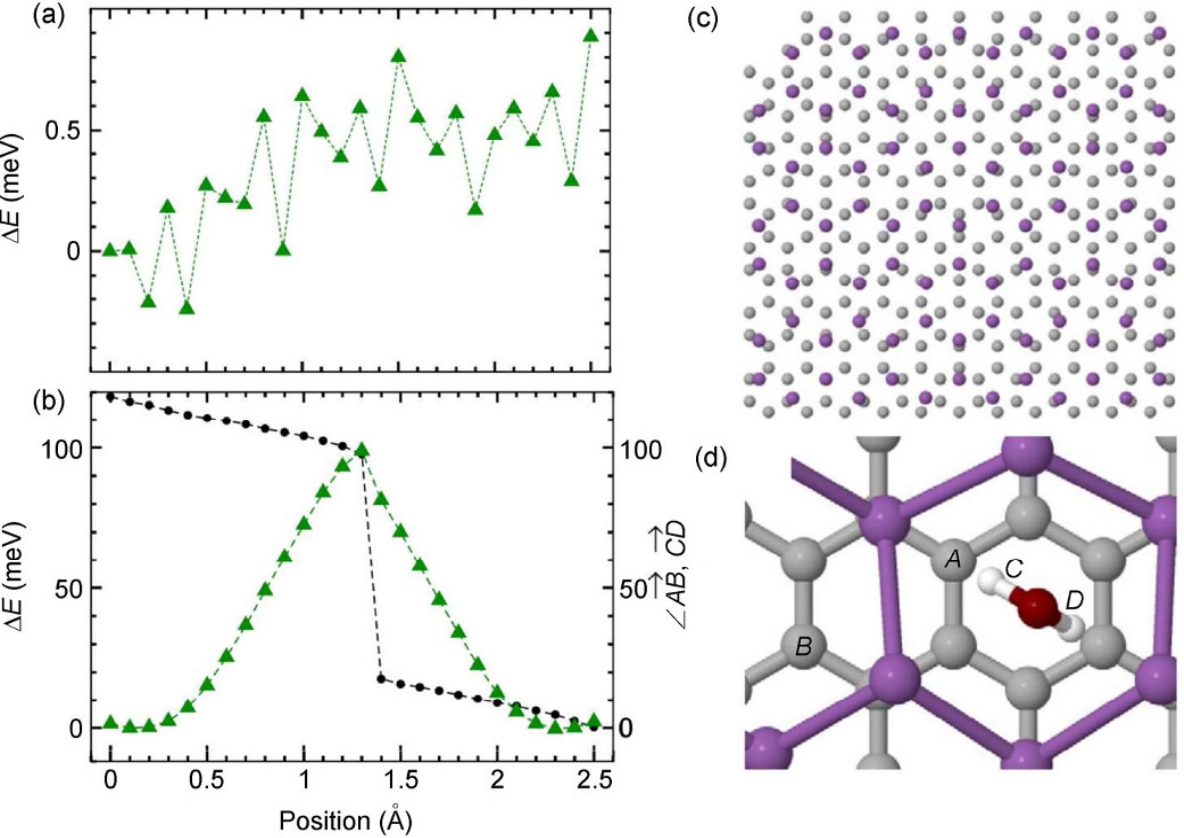

Fig. 17 DFT calculations of the potential energy landscape experienced by an antimony cluster sliding on top of an HOPG substrate. The small corrugation shown by the green triangles in (a) corresponds to the case of a clean, crystalline Sb/HOPG interface as shown (c) (grey: HOPG atoms, purple: Sb atoms). The larger corrugation shown by the green triangles in (b) is found for an $\mathrm{Sb} / \mathrm{HOPG}$ interface with a $\mathrm{H}_{2} \mathrm{O}$ molecule trapped in between (d). The black spheres in (b) indicate the relative orientation between the $\mathrm{H}_{2} \mathrm{O}$ molecule and the substrate during cluster movement. This shows that the trapped molecule is indeed mobile, which is a requirement to mediate friction between incommensurate interfaces (Reproduced with permission from Ref. [47], Copyright IOP Science, 2011). 
considering that the HOPG used for experiments was cleaved under ambient conditions [45]. On the other hand, a concentration of $1 \mathrm{Sb}_{4} / 7 \mathrm{~nm}^{2}$ was found to be required. While this concentration in itself seems to be very high, it might be understood by assuming that $\mathrm{Sb}_{4}$ clusters loosely attached to the sliding surface of the particle might act as "self-contaminants". In any case, these simulations affirm the plausibility of interface contamination to be responsible for the observed frictional duality.

\subsection{Scaling laws of structural lubricity}

The results described in the previous sections have already hinted at the potential of nanomanipulation experiments to analyze structural lubricity. In Fig. 15, a low friction branch was identified but a quantitative analysis of its contact area dependence has not yet been possible [45]. To address this issue, new experiments under improved conditions have been performed. The sensitivity of the optical detection system has been enhanced while at the same time also the particle preparation procedure was improved. Care was taken to avoid substrate contamination during particle preparation as thoroughly as possible, and experiments were conducted exclusively under UHV conditions in a short time span of only a few days after sample preparation in order to avoid ageing of the interface.

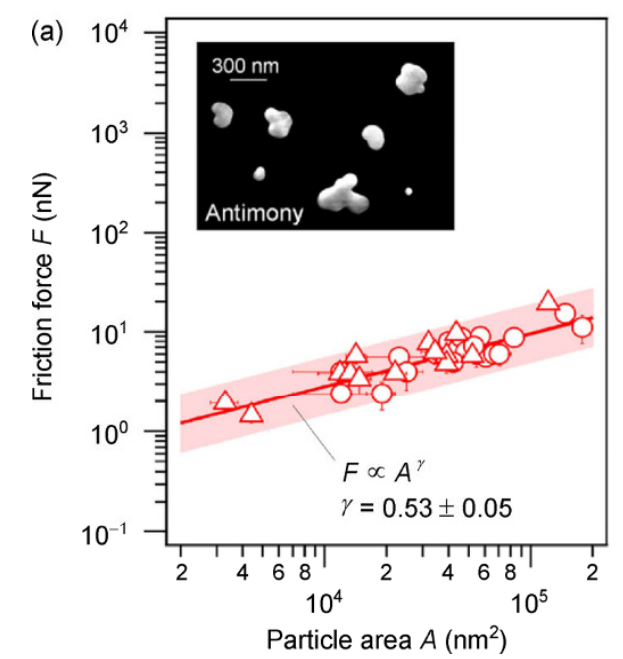

In addition to the antimony nanoparticles, which have already been analyzed previously, gold nanoparticles prepared by thermal evaporation were also studied, while HOPG was used as substrate for both materials. The choice of nanoparticles was motivated by their different crystalline structure. Round and compact antimony nanoparticles as they were used in Ref. [33] (cf., the inset of Fig. 18(a)) are expected to be of amorphous structure $[53,46]$. On the other hand, SEM measurements revealing triangular shapes for the gold nanoparticles clearly evidence crystallinity (inset of Fig. 18(b)). Topography images of the nanoparticles thus allow to directly determine the orientation of the particles, while the orientation of the substrate can be determined from atomically resolved stick-slip measurements in direct vicinity of the nanoparticles. The additional option to rotate the nanoparticles by off-center manipulations makes this sample system an ideal candidate to systematically analyze the directional dependence of interfacial friction in case of crystalline interfaces.

Interfacial friction has been measured for 32 antimony particles and 49 gold nanoparticles while the AFM was operated in contact mode for particle manipulation (see Section 2). Two independent sets of measurements have been carried out for each material to ensure reproducibility. Figure 18 shows the resulting contact area dependence of friction for

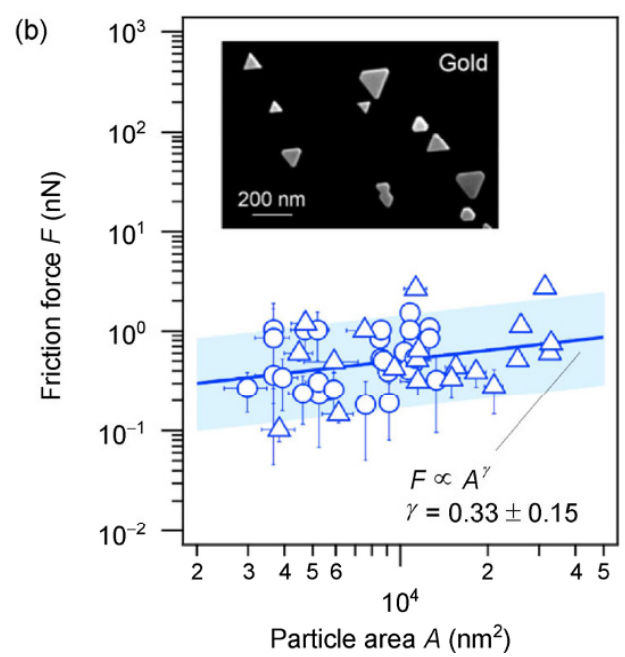

Fig. 18 Contact area dependence of friction measured during nanomanipulation experiments of $\mathrm{Sb}$ nanoparticles (a) and Au nanoparticles (b) on HOPG. The contact area dependence of the interfacial friction force $F$ has been fitted using a power law $F \propto A^{\gamma}$. In both cases, the values found for $\gamma$ are significantly smaller than 1, i.e., friction increases sublinearly with contact area. SEM images of typical $\mathrm{Sb}$ and $\mathrm{Au}$ nanoparticles are shown as insets. 
both the antimony (a) and the gold particles (b) in a double-logarithmic representation [33]. In both cases, the data shows the expected sublinear increase of fricton with particle size. To quantify the dependence, we fitted both data sets using the following power law:

$$
F_{\text {Friction }}=F_{0} \cdot A_{\text {contact }}^{\gamma}
$$

For the antimony nanoparticles an exponent of $\gamma_{\mathrm{Sb}}$ $=0.53 \pm 0.05$ is found, while the data measured for the gold nanoparticles results in an exponent of $\gamma_{\mathrm{Au}}=$ $0.34 \pm 0.15$. We also note that the larger error bars can only be partially attributed to the generally lower friction values. To understand this contact area dependence in more detail, let us revisit the considerations regarding the scaling behaviour related to structural lubricity first.

In case of an amorphous interface structure, symmetry arguments suggest that the contact area $A_{\text {contact }}$ should be the only parameter determining the scaling of interfacial friction [32], meaning that the interfacial friction is not affected by altering the particle's shape, orientation or direction of movement relative to the substrate as long as the contact area remains constant. Statistical arguments then lead to a scaling factor of $\gamma=0.5$, as discussed earlier in Section $3.1[32,82,81]$. This value is obviously in good agreement with the value that was actually found in our experiments, thereby validating the assumptions and conclusions of the theory as well as confirming the high quality of the experimental data.

For crystalline interfaces, however, the situation is more complex. In recent theoretical studies, de Wijn [32] has analytically calculated the friction for the case of triangular nanocrystals on a hexagonal substrate, a configuration suitable to describe, e.g., gold nanoparticles on HOPG. It is found that the scaling of friction with contact area sensitively depends on the particle's shape and orientation. For triangular particles with non-matching lattice constants, incommensurate and pseudocommensurate orientations have been identified, which result in different power laws describing the friction vs. contact area. While the friction scales $F_{\text {friction }} \propto A^{0}$ for incommensurate orienttations, an increase of friction described by $F_{\text {friction }} \propto$ $A^{0.5}$ is found for the pseudo-commensurate case.
The behaviour of irregular particles (e.g., round particles) is, however, even more difficult to describe by analytical arguments. To assess the influence of irregular particle shapes in more detail, we have therefore performed numerical simulations [96]. Clusters of antimony or gold atoms were placed on an HOPG substrate and the effective energy barrier of the particle was calculated for triangular and round particles based on realistic assumptions for the Lennard-Jones-Potential derived from experimental force-distance curves obtained by force spectroscopy on HOPG [97]. The results of these simulations are shown in Fig. 19, where the energy barrier of the particles has been normalized by the energy barrier found for a single atom. For gold nanoparticles of triangular shape, incommensurate and pseudocommensurate orientations need to be distinguished. While the incommensurate orientation results in $\gamma_{\mathrm{Au} \text {,incomm }} \approx 0$, pseudocommensurate orientations result in $\gamma_{\text {Au,pseudocomm }} \approx 0.45$; both values are in good agreement with the earlier analytical findings [32]. For the round gold nanoparticle an exponent of $\gamma_{\mathrm{Au} \text {,round }} \approx 0.25$ was found, a result that was almost independent of the particle orientation. This indicates that an irregular particle shape (i.e., a shape deviating from basic triangular form) does significantly influence the effects of both structural lubricity and pseudocommensurability.

To reconcile these concepts of scaling in the case of structural lubricity with the experimental results, Fig. 20 summarizes the different scaling laws in comparison with the experimental data. To make the sublinear increase evident, the friction data of Fig. 18 has been normalized by the friction of a single atom

$$
F_{\text {Friction,atom }}=\frac{\Delta E}{a}
$$

with $\Delta E$ representing the diffusion energy barrier of either gold on HOPG [98] or Sb on HOPG [53] and $a$ the distance between the hollow sites of HOPG. For an easier comparison between gold nanoparticles, antimony nanoparticles and theory, all data is plotted vs. the number of interface atoms [96]. We again find that the experimental data obtained for the antimony nanoparticle is in good agreement with the power law expected for amorphous particles, i.e., $\gamma_{\text {amorph,theo }}=$ 0.5 , but at the same time, it becomes evident that the 

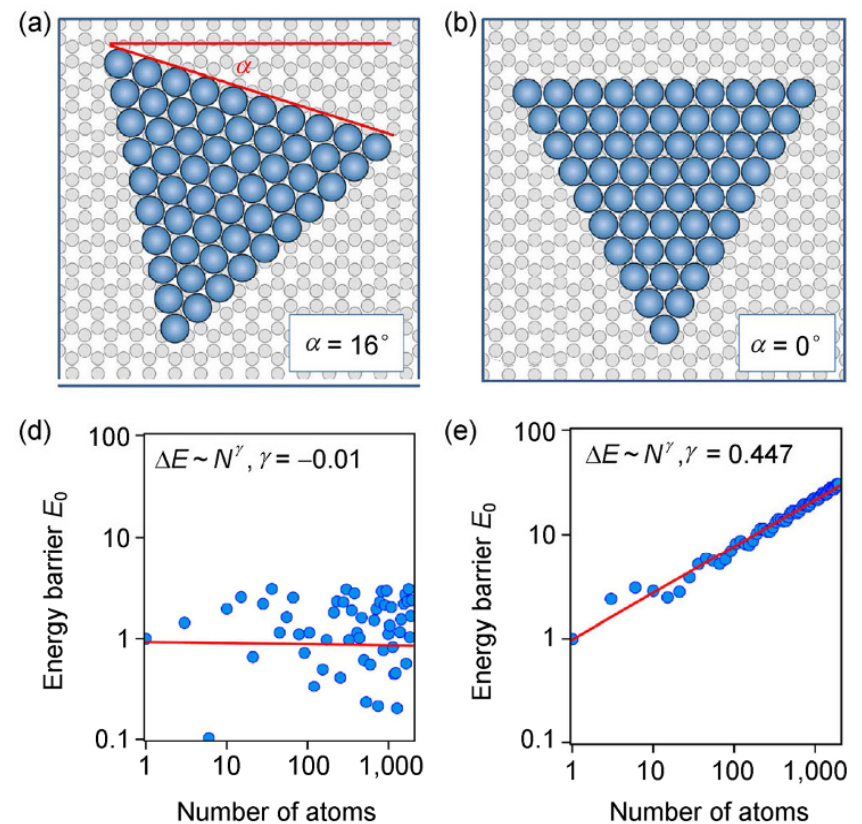
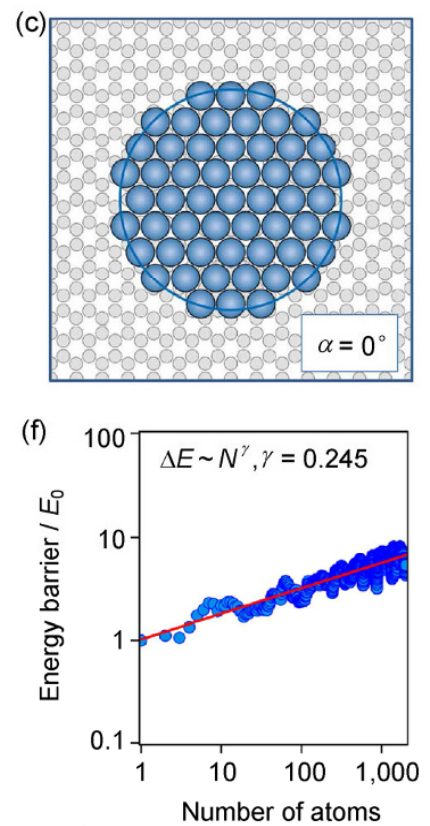

Fig. 19 Simulation results of particle vs. area scaling for triangular and round gold nanoparticles on HOPG. (a)-(c) illustrate the geometrical configurations, while (d)-(f) show the normalized energy barriers as a function of particle size (i.e., number of atoms). An angle $\alpha=16^{\circ}$ (a) corresponds to an incommensurate particle orientation, while an angle $\alpha=0^{\circ}$ (b) reflects a pseudocommensurate particle orientation. All simulation results could well be described by a power law $\Delta E \propto N^{\gamma}$ (d)-(f). Note that for triangular particles only atom configurations resulting in straight edges of the triangle have been taken into account. For round nanoparticles such a distinction could not be made, leading to a considerably higher number of data points (Reproduced with permission from Ref. [96], Copyright APS, 2013).

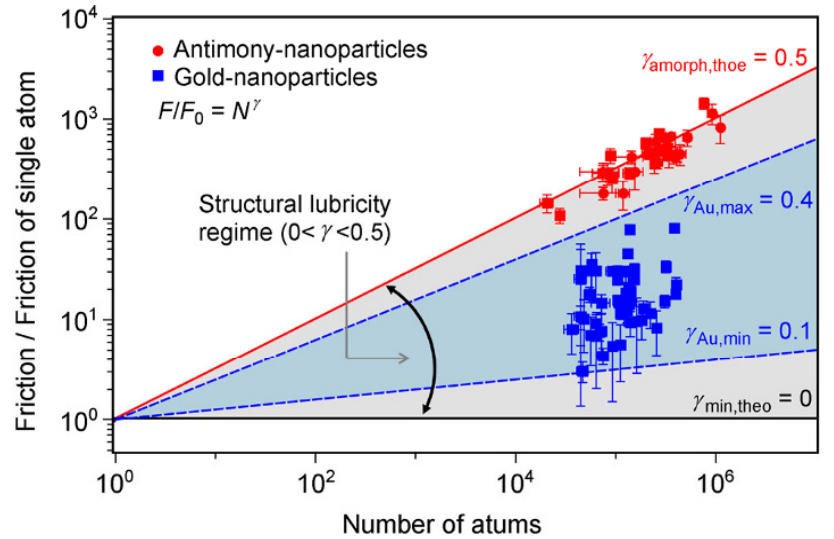

Fig. 20 Sublinear friction scaling laws compared with normalized friction data from particle sliding experiments. The friction $F$ is normalized by the single atom friction $F_{0}=\Delta E / a$ and the particle area by the atomic density to produce the number of interface atoms. In such a diagram the scaling law for structural lubricty becomes independent of those material parameters $\left(F / F_{0}=N^{\prime}\right)$. The normalized data points for antimony (red markers, using $\Delta E=$ $20 \mathrm{meV}$ for normalization) fall on the $\gamma=0.5$ line (red solid line). The normalized gold data (blue markers, $\Delta E=50 \mathrm{meV}$ ) is expected to obey different powers depending on shape and orientation and thus falls into a broader area confined by $\gamma=0.1$ and $\gamma=0.4$ (blue shading), which is well within the area marked as structural lubricity regime (Reproduced with permission from Ref. [33], Copyright APS, 2013). resulting curve also marks the upper limit of the range that is expected for any case of structural lubricity, as it coincides with the scaling expected for triangular gold nanoparticles under pseudo-commensurate orientation. The lower limit for the range of structural lubricity on the other hand is marked by $\gamma_{\min , \text { theo }}=0$, found for triangular gold nanoparticles under incommensurate orientation. And indeed, the friction values measured for gold nanoparticles are well within that range. More specifically, upper and lower limits of $\gamma_{\mathrm{Au} \text {,max }}=0.4$ and $\gamma_{\mathrm{Au} \text {,min }}=0.1$ can be determined from the experimental data. The scattering of the gold data is consistent with the influence of particle shape and orientation on friction. Arbitrarily shaped particle such as a hexagon with rounded edges, as it can be observed in the SEM images, may scale with any factor between zero and one half. But also perfectly shaped particles in the narrow transition range between commensurate and incommensurate orientations may show factors between zero and one half. More generally, the fact that no particles with $\gamma_{\mathrm{Au}}<0.1$ or $\gamma_{\mathrm{Au}}>0.4$ are found during the experiments might be attributed to the fact that realistic 
nanocrystals will always show some degree of imperfection.

To conclude, the presented experiments verify the theoretically predicted sublinear friction-area scaling power law for disordered surfaces. Owing to shape and orientation effects, the results for crystalline incommensurate interfaces fall into a range of power laws that is consistent with sublinear scaling as well. This allows to provide a link between mesoscopic friction and single atom diffusion processes. Thereby, the bridging of almost six orders of magnitude in scale represents a notable step towards one of the foremost goals of nanotribological research: to link molecular effects with mesoscopic and, ultimately, macroscopic behavior.

\section{Conclusion and outlook}

In recent years, analyzing friction by nanoparticle manipulation has become a field of growing interest in nanotribology. The quantitative extraction of interfacial friction from particle manipulation experiments opens the door for the analysis of many current issues in the field of nanoscale friction. Compared to conventional fricton force microscopy, the accessible range of materials is greatly enhanced and only limited by the ability to create nanoscale particles on flat surfaces. Due to the well-defined and clean interface conditions that can be achieved during nanoparticle manipulation experiments under UHV conditions, it is possible to verify fundamental tribological theories describing the friction between extended nanocontacts.

Most prominently, the contact area dependence of friction was analyzed in detail, a problem where experimental analysis was previously hindered by the geometric limitations of tip-sample contacts in conventional friction force microscopy. Analyzing metallic nanoparticles prepared on HOPG allowed to comfirm theoretically anticipated scaling laws for strucutral lubricity. Unique sublinear friction vs. contact area relations were found and could be related to the specific structures of the nanoparticles. Experiments also showed that nanoparticle manipulation strategies are also suitable to approach other current issues in nanotribology like the influence of interface contamination on friction.
Over the years, different experimental strategies have evolved, the choice of which mostly depends on the experimental conditions or the nanoparticles to be analyzed. Nowadays, especially the "tip on top" approach seems to be very promising for the analysis of fundamental friction processes. It was initially introduced to measure the difference between static and sliding friction, but the permanent contact between tip and nanoparticle also makes it an interesting approach to perform systematic measurements with, e.g., variation of the normal force or sliding velocity. Through this approach, it can be analyzed how essential concepts describing atomic friction, such as contact ageing or the thermally activated PrandtlTomlinson-Model, can be transferred to extended nanocontacts.

Finally, let us point out that the analysis of friction by nanoparticle manipulations is not only interesting for analyzing fundamental friction laws, but can also be relevant for technological applications. Compared to conventional friction force microscopy with contact sizes of about $10-100 \mathrm{~nm}^{2}$, nanoparticle manipulation allows the analysis of far more realistic contact sizes, as they are, e.g., be found in micro- or nanoelctromechanical systems (MEMS, NEMS) where friction and wear currently still limit the perspective for widespread application. Understanding friction for such mesoscale contacts is therefore expected to have a considerable impact on technological applications.

\section{Acknowledgements}

Financial support was provided by the DFG (Project SCHI 619/8-1), the EUROCORES program FANAS of the European Science Foundation, and the EC 6th framework program (Grant No. ERAS-CT-2003980409).

U. S. acknowledges primary financial support by the National Science Foundation through the Yale Materials Research Science and Engineering Center (Grant No. MRSEC DMR-1119826).

Open Access: This article is distributed under the terms of the Creative Commons Attribution License which permits any use, distribution, and reproduction in any medium, provided the original author(s) and source are credited. 


\section{References}

[1] Craighead H G. Nanoelectromechanical systems. Science 290: 1532-1535 (2000)

[2] Ekinci K L, Roukes M L. Nanoelectromechanical systems. Review of Scientific Instruments 76: 061101 (2005)

[3] Handbook of Micro/Nanotribology. Bhushan B, Ed. CRC Press LLC, 1999.

[4] Bhushan B, Israelachvili J N, Landman U. Nanotribology: Friction, wear and lubrication at the atomic scale. Nature 374: 607-616 (1995)

[5] Mate M, McClelland G M,Erlandsson R, Chiang S. Atomicscale friction of a Tungsten tip on a graphite surface. Phys Rev Lett 59: 1942-1946 (1987)

[6] Binnig G, Quate C F, Gerber C. Atomic force microscope. Phys Rev Lett 56: 930-933 (1986)

[7] Carpick R W, Salmeron M. Scratching the surface: Fundamental investigations of tribology with atomic force microscopy. Chem Rev 97: 1163-1194 (1997)

[8] Fundamentals of Friciton and Wear on the Nanoscale. Gnecco E, Meyer E, Ed. Berlin: Springer, 2007.

[9] Bowden F P, Tabor D. Friction and Lubrication of Solids. Oxford(UK): Oxford University Press, 1950.

[10] Holscher H, Schirmeisen A, Schwarz U. D. Principles of atomic friction: from sticking atoms to superlubric sliding. Philosophical Transactions of the Royal Society A: Mathematical, Physical and Engineering Sciences 366: 1383-1404 (2008)

[11] Carpick R W, Agraït N, Ogletree D F, Salmeron M. Measurement of interfacial shear (friction) with an ultrahigh vacuum atomic force microscope. J Vac Sci Technol B 14: 1289 (1996)

[12] Lantz M A, O'Shea S J, Welland M E, Johnson K L. Atomic-force-microscope study of contact area and friction on $\mathrm{NbSe}_{2}$. Phys Rev B 55: 10776 (1997)

[13] Schwarz U D, Zwörner O, Köster P, Wiesendanger R. Quantitative analysis of the frictional properties of solid materials at low loads. I. Carbon compounds. Phys Rev B 56: 6987 (1997)

[14] Schwarz U D, Zwörner O, Köster P, Wiesendanger R. Quantitative analysis of the frictional properties of solid materials at low loads. II. Mica and germanium sulfide. Phys Rev B 56: 6997 (1997).

[15] Meyer E, Lüthi R, Howald L, Bammerlin M, Guggisberg M, Güntherodt H-J. Site-specific friction force spectroscopy. J Vac Sci Technol B 14: 1285 (1996)

[16] Enachescu M, van den Oetelaar R J A, Carpick R W, Ogletree D F, Flipse C F J, Salmeron M. Atomic force microscopy study of an ideally hard contact: The diamond (111)/tungsten carbide interface. Phys Rev Lett 81: 1877 (1998)

[17] Gnecco E, Bennewitz R, Gyalog T, Loppacher Ch, Bammerlin M, Meyer E, Güntherodt H-J. Velocity dependence of atomic friction. Phys Rev Lett 84: 11721175 (2000)

[18] Evstigneev M, Schirmeisen A, Jansen L, Fuchs H, Reimann P. Force dependence of transition rates in atomic friction. Phys Rev Lett 97: 240601 (2006)

[19] Zwörner O, Hölscher H, Schwarz U D, Wiesendanger R. The velocity dependence of frictional forces in point-contact friction. Appl Phys A 66: S263-267 (1998)

[20] Jansen L, Hölscher H, Fuchs H, Schirmeisen A. Temperature dependence of atomic-scale stick-slip friction. Phys Rev Lett 104: 256101 (2010)

[21] Schirmeisen A, Jansen L, Hölscher H, Fuchs H. Temperature dependence of point contact friction on silicon. Appl Phys Lett 88: 123108 (2006)

[22] Zhao X, Hamilton M, Sawyer W G, Perry S S. Thermally activated friction. Trib Lett 27: 113-117 (2007)

[23] Barel I, Urbakh M, Jansen L, Schirmeisen A. Multibond dynamics of nanoscale friction: The role of temperature. Phys Rev Lett 104: 066104 (2010)

[24] Overney R M, Takano H, Fujihira M, Paulus W, Ringsdorf H. Ansiotropy in friction and molecular stick-slip motion. Phys Rev Lett 72: 3546 (1994)

[25] Bluhm H, Schwarz U D, Meyer K P, Wiesendanger R. Anisotropy of sliding friction on the triglycine sulfate (010) surface. Appl Phys A 61: 525 (1995)

[26] Shindo H, Shitagami K, Sugai T, Kondo S-I. Evidence of the contribution of molecular orientations on the surface force friction of alkaline earth sulfate crystals. Phys Chem Chem Phys 1: 1597-1600 (1995)

[27] Park J Y, Ogletree D F, Salmeron M, Ribeiro R A, Canfield P C, Jenks C J, Thiel P A. High frictional anisotropy of periodic and aperiodic directions on a quasicrystal surface. Science 309: 1354-1356 (2005)

[28] Meyer E, Overney R, Brodbeck D, Howald L, Lüthi R, Frommer J, Güntherodt H-J. Friction and wear of LangmuirBlodgett films observed by friction force microscopy. Phys Rev Lett 69: 1777 (1992)

[29] Overney R M, Meyer E, Frommer J, Brodbeck D, Lüthi R, Howald L, Güntherodt H-J, Fujihira M, Takano H, Gotoh Y. Friction measurements on phase-separated thin films with a modified atomic force microscope. Nature 359: 133 (1992)

[30] Schwarz U D, Allers W, Gensterblum G, Wiesendanger R. Low-load friction behavior of epitaxial $\mathrm{C}_{60}$ monolayers under Hertzian contact. Phys Rev B 52: 14976 (1995) 
[31] Dienwiebel M, Verhoeven G S, Pradeep N, Frenken J W M, Heimberg J A, Zandbergen H W. Superlubricity of graphite. Phys Rev Lett 92: 126101 (2004)

[32] de Wijn A S. (In)commensurability, scaling, and multiplicity of friction in nanocrystals and application to gold nanocrystals on graphite. Phys Rev B 86: 085429 (2012)

[33] Dietzel D, Feldmann M, Fuchs H, Schwarz U D, Schirmeisen A. Scaling laws of structural lubricity. Phys Rev Lett 111: 235502 (2013)

[34] Israelachvili J N , Tabor D. Shear properties of molecular films. Wear 24: 386-390 (1973)

[35] Briscoe B J, Evans D C B. The shear properties of LangmuirBlodgett layers. Proc Roy Soc Lond A 380: 389-407 (1982)

[36] Krim J, Widom A. Damping of a crystal oscillator by an adsorbed monolayer and its relation to interfacial viscosity. Phys Rev B 38: 12184 (1988)

[37] Krim J, Solina D H, Chiarello R. Nanotribology of a $\mathrm{Kr}$ monolayer: A quartz-crystal microbalance study of atomicscale friction. Phys Rev Lett 66: 181-184 (1991)

[38] Coffey T, Krim J. Impact of substrate corrugation on the sliding friction levels of adsorbed films. Phys Rev Lett 95 : 076101 (2005)

[39] Lüthi R, Meyer E, Haefke H, Howald L, Gutmannsbauer W, Güntherodt H-J. Sled-type motion on the nanometer scale: Determination of dissipation and cohesive energies of C60. Science 266: 1979-1981 (1994)

[40] Sheehan P E, Lieber C M. Nanotribology and nanofabrication of $\mathrm{MoO}_{3}$ structures by atomic force microscopy. Science 271: 1158-1161 (1996)

[41] Falvo M R, Steele J, Taylor II R M, Superfine R. Gearlike rolling motion mediated by commensurate contact: Carbon nanotubes on HOPG. Phys Rev B 62: R10665 (2000)

[42] Rao A, Gnecco E, Marchetto D, Mougin K, Schönenberger M, Valeri S, Meyer E. The analytical relations between particles and probe trajectories in atomic force microscope nanomanipulation. Nanotechnology 20: 115706 (2009)

[43] Polyakov B, Vlassov S, Dorogin L M, Butikova J, Antsov M, Oras S, Lohmus R, Kink I. Manipulation of nanoparticles of different shapes inside a scanning electron microscope. Beilstein Journal of Nanotechnology 5: 133-140 (2014)

[44] Mougin K, Gnecco E, Rao A, Cuberes M T, Jayaraman S, McFarland E W, Haidara H, Meyer E. Manipulation of gold nanoparticles: Influence of surface chemistry, temperature, and environment (vacuum versus ambient atmosphere). Langmuir 24: 1577-1581 (2008)

[45] Dietzel D, Ritter C, Mönninghoff T, Fuchs H, Schirmeisen A, Schwarz U D. Frictional duality observed during nanoparticle sliding. Phys Rev Lett 101: 125505 (2008)

[46] Dietzel D, Mönninghoff T, Herding C, Feldmann M, Fuchs
H, Stegemann B, Ritter C, Schwarz U D, Schirmeisen A. Frictional duality of metallic nanoparticles: Influence of particle morphology, orientation, and air exposure. Phys Rev B 82: 035401 (2010)

[47] Brndiar J, Turansky R, Dietzel D, Schirmeisen A, Stich I. Understanding frictional duality and bi-duality: Sbnanoparticles on HOPG. Nanotechnology 22: 085704 (2011)

[48] Ritter C, Heyde M, Stegemann B, Rademann K, Schwarz U D. Contact-area dependence of frictional forces: Moving adsorbed antimony nanoparticles. Phys Rev B 71: 085405 (2005)

[49] Paolicelli G, Rovatti M, Vanossi A, Valeri S. Controlling single cluster dynamics at the nanoscale. Appl Phys Lett 95: 143121 (2009)

[50] Polyakov B, Dorogin L M, Vlassov S, Kink I, Romanov A E, Lohmus R. Simultaneous measurement of static and kinetic friction of $\mathrm{ZnO}$ nanowires in situ with a scanning electron microscope. Micron 43: 1140-1146 (2012)

[51] Dietzel D, Feldmann M, Fuchs H, Schwarz U D, Schirmeisen A. Transition from static to kinetic friction of metallic nanoparticles. Appl Phys Lett 95: 053104 (2009)

[52] Feldmann M, Dietzel D, Schwarz U D, Fuchs H, Schirmeisen A. Influence of contact aging on nanoparticle friction kinetics. Phys Rev Lett 112: 155503 (2014)

[53] Stegemann B, Ritter C,Kaiser B, Rademann K. Crystallization of antimony nanoparticles: Pattern formation and fractal growth. J Phys Chem B 108: 14292-14297 (2004)

[54] Kaiser B, Stegemann B, Kaukel H, Rademann K. Instabilities and pattern formation during the self-organized growth of nanoparticles on graphite. Surf Sci 496: L18-L22 (2002)

[55] Ritter C, Baykara M Z, Stegemann B, Heyde M, Rademann K, Schroers J, Schwarz U D. Nonuniform friction-area dependency for antimony oxide surfaces sliding on graphite. Phys Rev B 88: 045422 (2013)

[56] Tranvouez E, Orieux A, Boer-Duchemin, C. H. Devillers E, Huc V, Comtet G, Dujardin G. Manipulation of cadmium selenide nanorods with an atomic force microscope. Nanotechnology 20: 165304 (2009)

[57] Bombis Ch, Ample F, Mielke J, Mannsberger M, Villagmez C J, Roth Ch, Joachim C, Grill L. Mechanical behavior of nanocrystalline $\mathrm{NaCl}$ islands on $\mathrm{Cu}(111)$. Phys Rev Lett 104: 185502 (2010)

[58] Feng X, Kwon S, Park J Y, Salmeron M. Superlubric sliding of graphene nanoflakes on graphene. ACS Nano 7: 1718-1724 (2013)

[59] Zhong Q, Inniss D, Kjoller K, Elings V. Fractured polymer/ silica fiber surface studied by tapping mode atomic force microscopy. Surf Sci Lett 290: L688 (1993) 
[60] Albrecht T R, Grütter P, Horne D, Rugar D. Frequency modulation detection using high- $Q$ cantilevers for enhanced force microscope sensitivity. J Appl Phys 69: 668 (1991)

[61] Ritter C, Heyde M, Schwarz U D, Rademann K. Controlled translational manipulation of small latex spheres by dynamic force microscopy. Langmuir 18: 7798-7803 (2002)

[62] Anczykowski B, Gotsmann B, Fuchs H, Cleveland J P, Elings V B. How to measure energy dissipation in dynamic mode atomic force microscopy. Appl Surf Sci 140: 376-382 (1999)

[63] Aruliah D A, Müser M, Schwarz U D. Calculations of the threshold force and threshold power to move adsorbed nanoparticles. Phys Rev B 71: 085406 (2005)

[64] Schwarz U D, Ritter C, Heyde M. Nanotribological studies by nanoparticle manipulation. In Fundamentals of Friction and Wear on the Nanoscale. Meyer E, Gnecco E, Ed. Heidelberg: Springer, 2007: 561-582.

[65] Darwich S, Mougin K, Rao A, Gnecco E, Jayaraman S, Haidara H. Manipulation of gold colloidal nanoparticles with atomic force microscopy in dynamic mode: Influence of particle substrate chemistry and morphology, and of operating conditions. Beilstein Journal of Nanotechnology 2: 85-98 (2011)

[66] Tripathi M, Paolicelli G, D“Addato S, Valerie S. Controlled AFM detachments and movement of nanoparticles: Gold clusters on HOPG at different temperatures. Nanotechnology 23: 245706 (2012)

[67] Ternes M, Lutz C P, Hirjibehedin C F, Giessibl F J, Heinrich A J. The force needed to move an atom on a surface. Science 319: 1066 (2008)

[68] Langewisch G, Falter J, Fuchs H, Schirmeisen A. Forces during the controlled displacement of organic molecules. Phys Rev Lett 110: 036101 (2013)

[69] Palacio M, Bhushan B. A nanoscale friction investigation during the manipulation of nanoparticles in controlled environments. Nanotechnology 19: 315710 (2008)

[70] Dietzel D, Mönninghoff T, Jansen L, Fuchs H, Ritter C, Schwarz U D, Schirmeisen A. Interfacial friction obtained by lateral manipulation of nanoparticles using atomic force microscopy techniques. J Appl Phys 102: 084306 (2007)

[71] Ueyama H, Sugawara Y, Morita S. Stable operation mode for dynamic noncontact atomic force microscopy. Appl Phys A Mater Sci Process 66: 295 (1998)

[72] Schirmeisen A, Hölscher H, Anczykowski B, Weiner D, Schäfer M M, Fuchs H. Dynamic force spectroscopy using the constant-excitation and constant-amplitude modes. Nanotechnology 16: S13 (2005)
[73] Dietzel D, Feldmann M, Herding C, Schwarz U D, Schirmeisen A. Quantifying pathways and friction of nanoparticles during controlled manipulation by contactmode atomic force microscopy. Trib Lett 39: 273-281 (2010)

[74] Rao A, Wille M L, Gnecco E, Mougin K, Meyer E. Trajectory fluctuations accompanying the manipulation of spherical nanoparticles. Phys Rev B 80: 193405 (2009)

[75] Nita P, Casado S, Dietzel D, Schirmeisen A, Gnecco E. Spinning and translational motion of $\mathrm{Sb}$ nanoislands manipulated on $\mathrm{MoS}_{2}$. Nanotechnology 24: 325302 (2013)

[76] Liu Y, Szlufarska I. Chemical origins of frictional aging. Phys Rev Lett 109: 186102 (2012)

[77] Li Q, Tullis T E, Goldsby D E, Carpick R W. Frictional ageing from interfacial bonding and the origins of rate and state friction. Nature 480: 233 (2011)

[78] Evstvigeneev M, Reimann P. Thermally activated contact strengthening explains nonmonotonic temperature and velocity dependence of atomic friction. Phys $\operatorname{Rev} X$ 3: 041020 (2013)

[79] Persson B N J. Theory and simulation of sliding friction. Phys Rev Lett 71: 1212 (1993)

[80] Greenwood J A, Williamson J B P. Contact of nominally flat surfaces. Proc R Soc Lond A 295: 300 (1966)

[81] Müser M. Theoretical aspects of superlubricity. In Fundamentals of Friction and Wear on the Nanoscale. Meyer E, Gnecco E, Ed. Heidelberg: Springer, 2007: 177-210.

[82] Müser M H, Wenning L, Robbins M O. Simple microscopic theory of Amontons's laws for static friction. Phys Rev Lett 86: 1295-1298 (2001)

[83] Hirano M, Shinjo K. Atomistic locking and friction. Phys Rev B 41: 11837 (1990)

[84] Shinjo K, Hirano M. Dynamics of friction: Superlubric state. Surf Sci 283: 473-478 (1993)

[85] Müser M H. Structural lubricity: Role of dimension and symmetry. Europhys Lett 66: 97-103 (2004)

[86] Martin J M, Donnet C, Mogne T L, Epicier T. Superlubricity of molybdenum disulphide. Phys Rev B 48: 10583 (1993)

[87] Hirano M, Shinjo K, Kaneko R, Murata Y. Observation of superlubricity by scanning tunneling microscopy. Phys Rev Lett 78: 1448 (1997)

[88] Crossley A, Kisi E H, Summers J W B, Myhra S. Ultra-low friction for a layered carbide-derived ceramic, $\mathrm{Ti}_{3} \mathrm{SiC}_{2}$, investigated by lateral force microscopy (LFM). J Phys $D$ 32: 632 (1999)

[89] Liu Z, , Yang J, Grey F, Liu J Z, Liu Y, Wang Y, Yang Y, Cheng Y, Zheng Q. Observation of microscale superlubricity in graphite. Phys Rev Lett 108: 205503 (2012) 
[90] Reguzzonia M, Ferrarioa M, Zapperia S, Righia M C. Onset of frictional slip by domain nucleation in adsorbed monolayers. PNAS 107: 1313 (2010)

[91] Hirano M, Shinjo K. Superlubricity and frictional anisotropy. Wear 168: 121-125 (1993)

[92] Sørensen M R, Jacobsen K W, Stoltze P. Simulations of atomic-scale sliding friction. Phys Rev B 53: 2101-2113 (1996)

[93] Müser M H, Robbins M O. Conditions for static friction between flat crystalline surfaces. Phys Rev B 61: 2335-2342 (2000)

[94] Mo Y, Turner K T, Szlufarska I. Friction laws at the nanoscale. Nature 457: 1116-1119 (2009)

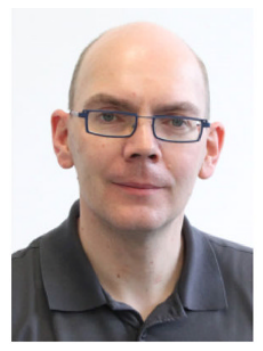

Dirk DIETZEL. He received his Diploma and $\mathrm{PhD}$ degree in Experimental Physics from the University of Bochum, Germany, in 1997 and 2001, respectively. After two years as a PostDoc at the University of Bordeaux, France, he joined the Nanomechanics Group

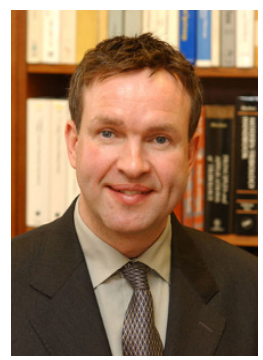

Udo D. SCHWARZ. He is a Professor of Mechanical Engineering \& Materials Science and Chemical \& Environmental Engineering, Yale University. He graduated in 1989 from the University of Basel, Switzerland, receiving his $\mathrm{PhD}$ degree in physics from the same institution in 1993. Subsequently, he continued his work as a staff scientist and lecturer at the Institute of Applied Physics of the University of Hamburg, Germany. In 2001, Prof. Schwarz moved
[95] He G, Müser M H, Robbins M O. Adsorbed layers and the origin of static friction. Science 284: 1650-1652 (1999)

[96] Dietzel D, Feldmann M, Fuchs H, Schwarz U D, Schirmeisen A. Scaling laws of structural lubricity-Supplemental material. Phys Rev Lett 111: 235502 (2013)

[97] Hölscher H, Allers W, Schwarz U D, Schwarz A, Wiesendanger R. Interpretation of true atomic resolution images of graphite (0001) in noncontact atomic force microscopy. Phys Rev B 62: 6967-6970 (2000)

[98] Jensen P, Blase X, Ordéjon P. First principles study of gold adsorption and diffusion on graphite. Surf Sci 564: 173-178 (2004)

of Andre Schirmeisen at the University of Münster, Germany. Since 2011 he is working at the University of Giessen, Germany, where he is heading the nanofriction branch at the Institute of Applied Physics. His main research areas are atomic force microscopy and nanotribology, with a particular focus on applying nanomanipulation techniques to tribological questions.

to the Materials Science Department of the Lawrence Berkeley National Laboratory in Berkeley, California. Since 2002, he works at Yale's Mechanical Engineering Department, where he got promoted to full professor in 2009 and department chair in 2012. His research interests concern the local measurement of atomicscale interactions and properties, including electric, magnetic, chemical, and nanotribological interactions, using scanning probe microscopy techniques to study problems in surface physics, catalysis, friction, and adhesion. 


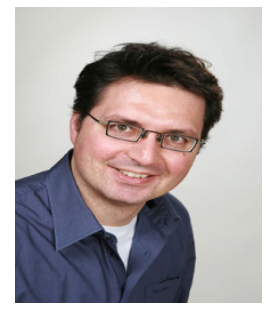

André SCHIRMEISEN. $\mathrm{He}$ is a Professor and Director of the Institute of Applied Physics (IAP), Justus-Liebig University Giessen, Giessen, Germany. He graduated in 1995 from the RWTH Aachen, Germany and received his $\mathrm{PhD}$ degree in physics from McGill University, Montréal, Canada in 1999. After this he worked as a strategic business consultant for the Boston Consulting Group for a year and then re-continued his academic career in
2001 as a staff scientist and lecturer at the Institute of Physics at the Westfalian-Wilhelms University Münster, Germany. In 2010 he accepted a senior professorship position at the Institute of Applied Physics at the Justus-Liebig University Giessen. His research interests concern the local measurement of atomic-scale force interactions, with a special focus on nano- and mesoscopic tribology phenomena. Further interests are self-assembly mechanisms of functional organic molecules on surfaces as well as local conductivity properties of battery electrodes and ionic liquids. 